\title{
Autologous mesenchymal stem cell-derived dopaminergic neurons function in parkinsonian macaques
}

\author{
Takuya Hayashi, 1,2,3,4 Shohei Wakao,5 Masaaki Kitada,5 Takayuki Ose,, ${ }^{1}$ Hiroshi Watabe,1,6 \\ Yasumasa Kuroda,7 Kanae Mitsunaga, 5 Dai Matsuse,,5 Taeko Shigemoto, 5 Akihito Ito,8 \\ Hironobu Ikeda, ${ }^{8}$ Hidenao Fukuyama, ${ }^{3}$ Hirotaka Onoe, ${ }^{1}$ Yasuhiko Tabata, ${ }^{9}$ and Mari Dezawa ${ }^{5,7}$ \\ ${ }^{1}$ Functional Probe Research Laboratory, Center for Molecular Imaging Science, RIKEN, Kobe, Japan \\ ${ }^{2}$ National Cerebral Cardiovascular Center Research Institute, Osaka, Japan. ${ }^{3}$ Human Brain Research Center, Graduate School of Medicine, and \\ ${ }^{4}$ Center for iPS Cell Research and Application, Kyoto University, Kyoto, Japan. 5Department of Stem Cell Biology and Histology, \\ Tohoku University Graduate School of Medicine, Sendai, Miyagi, Japan. ${ }^{6}$ Faculty of Molecular Imaging in Medicine, \\ Osaka University Graduate School of Medicine, Suita, Osaka, Japan. 'Department of Anatomy and Anthropology, \\ Tohoku University Graduate School of Medicine, Sendai, Miyagi, Japan. ${ }^{8}$ Shiga Research Institute, Nissei Bilis Co. Ltd., Koga, Shiga, Japan. \\ ${ }^{9}$ Department of Biomaterials, Field of Tissue Engineering, Institute for Frontier Medical Sciences, Kyoto University, Kyoto, Japan.
}

\begin{abstract}
A cell-based therapy for the replacement of dopaminergic neurons has been a long-term goal in Parkinson's disease research. Here, we show that autologous engraftment of A9 dopaminergic neuron-like cells induced from mesenchymal stem cells (MSCs) leads to long-term survival of the cells and restoration of motor function in hemiparkinsonian macaques. Differentiated MSCs expressed markers of A9 dopaminergic neurons and released dopamine after depolarization in vitro. The differentiated autologous cells were engrafted in the affected portion of the striatum. Animals that received transplants showed modest and gradual improvements in motor behaviors. Positron emission tomography (PET) using $\left[{ }^{11} \mathrm{C}\right]-\mathrm{CFT}$, a ligand for the dopamine transporter (DAT), revealed a dramatic increase in DAT expression, with a subsequent exponential decline over a period of 7 months. Kinetic analysis of the PET findings revealed that DAT expression remained above baseline levels for over 7 months. Immunohistochemical evaluations at 9 months consistently demonstrated the existence of cells positive for DAT and other A9 dopaminergic neuron markers in the engrafted striatum. These data suggest that transplantation of differentiated autologous MSCs may represent a safe and effective cell therapy for Parkinson's disease.
\end{abstract}

\section{Introduction}

Cell-based therapies are expected to replace the missing dopaminergic neurons and to restore the motor function in patients with Parkinson's disease (PD) (1). Early studies on cell-based therapies used fetal midbrain tissue containing dopaminergic neurons as a cell source and suggested potential therapeutic effects in PD (for review, see refs. 2, 3). However, limited availability and ethical considerations relating to the use of fetuses pose limitations for practical use. Bone marrow-derived mesenchymal stem cells (MSCs), a type of adult stem cells, have trophic effects (4) and a differentiation spectrum that crosses oligolineage boundaries (5), offering the potential for use in autologous cell therapy, with low risk of tumorigenesis (6). The MSCs have been already tested for cell therapy in PD model rodents (7-9) and even in patients with PD (10). However, they have shown poor performance for restoration of motor function, potentially due to limited spontaneous differentiation (11) or facilitated apoptosis $(12,13)$ of MSCs. Recent studies of fetal midbrain graft have suggested that better outcomes could be obtained if the graft consisted of well-differentiated A9 dopaminergic neurons (14-16), the most severely damaged neuronal type in PD (17). Therefore, differentiation of MSCs into desired

Authorship note: Takuya Hayashi and Shohei Wakao contributed equally to this work.

Conflict of interest: The authors have declared that no conflict of interest exists. Citation for this article: J Clin Invest. 2013;123(1):272-284. doi:10.1172/JCI62516 cells, such as A9 dopaminergic neurons, would probably provide effective functional restoration in PD.

Recently, it was shown that MSCs could be artificially directed to differentiate into several specialized cell types, including those in nervous tissues (18-21). Previously, we reported that dopamine-producing cells could be induced from MSCs (MSC-DP cells) by introduction of a Notch1 intracellular domain-containing (NICD-containing) plasmid, followed by cytokine stimulation with bFGF, forskolin, ciliary neurotrophic factor (CNTF), and glial cell linederived neurotrophic factor (GDNF) $(20,21)$. The differentiated cells were positive for markers of dopaminergic neurons, such as tyrosine hydroxylase (TH) and the dopamine transporter (DAT), and had an ability to release dopamine after depolarization by potassium stimulation. When rat and human MSC-DP cells were transplanted into the striata of $\mathrm{PD}$ model rats, integration of $\mathrm{TH}^{+}$and $\mathrm{DAT}^{+}$cells and functional recovery in motor behaviors were confirmed (20). Subsequent development of a spermine-pullulan-mediated reverse transfection method allowed us to induce MSC-DP cells more safely and efficiently than before from MSCs of macaque monkeys (21), an animal species frequently used for preclinical trials of PD (22-27).

To test the scalability of MSC-DP cell-based therapy in primates in this study, monkey MSC-DP cells were characterized in detail using specific markers and evaluated for their longitudinal effects after they were engrafted into hemiparkinsonian monkeys using behavioral tests and positron emission tomography (PET). The MSC-DP cells, prepared autologously from the bone marrow of 

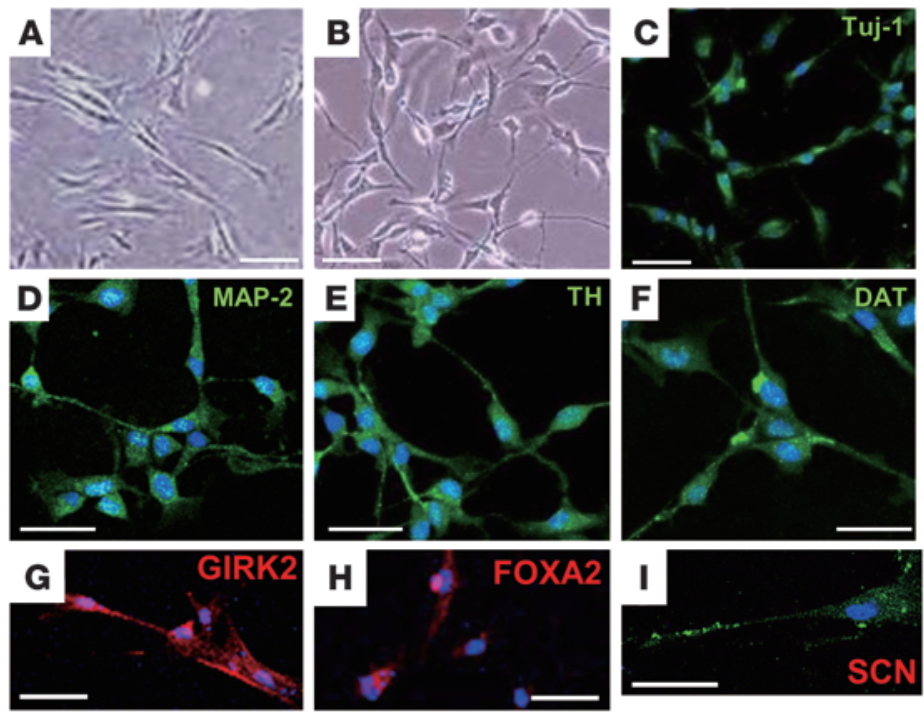

\section{Figure 1}

Monkey bone marrow MSCs and MSC-DP cells. (A) Morphological changes were evident in cynomolgus naive MSCs (phasecontrast microscopy) and (B) MSC-DP cells (phase-contrast microscopy). The MSC-DP cells possessed neurite-like processes. Immunocytochemistry of MSC-DP cells showed that the cells were immunoreactive for the neuronal markers (C) Tuj1 and (D) MAP-2; for the markers of dopaminergic neurons (E) TH, (F) DAT, (G) GIRK2, and (H) FOXA2; and for the marker of neurons, (I) sodium channel (SCN). DAPI was used for counterstaining of nuclei. Scale bar: $30 \mu \mathrm{m}$. (J) Results of RT-PCR in naive MSCs, MSC-DP cells, and tissue samples from the SNc and VTA. Naive MSCs expressed no GIRK2, FOXA2, and CALB1 mRNA, while the MSC-DP cells expressed GIRK2 and FOXA2 mRNA. SNc tissue samples from an embryo and an adult cynomolgus monkey also contained high levels of GIRK2 and FOXA2 mRNA but only low levels of CALB1 mRNA, while the VTA contained high levels of CALB1 mRNA and only very low levels of GIRK2 and FOXA2 mRNA. NC, negative control. (K) Percentages of cells immunoreactive for TH, DAT, GIRK2, and FOXA2 in MSC-DP cells.
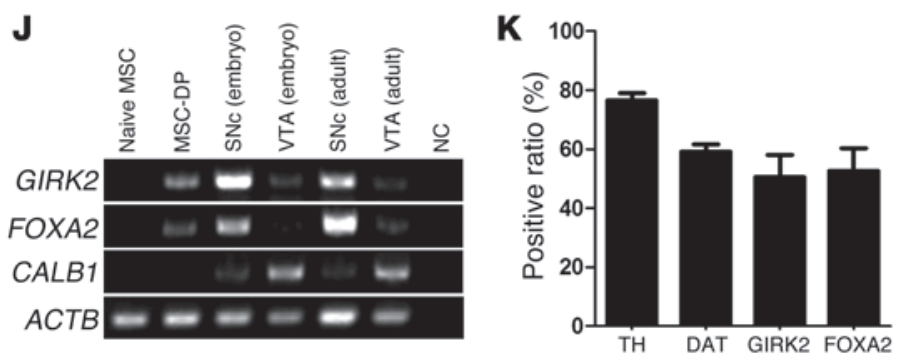

each test animal, expressed cell makers not only for antigens that have been previously described $(20,21)$, such as $\beta$ tubulin III (Tuj1), microtubule-associated protein 2 (MAP-2), TH, and DAT, but also for those specific to the A9 subtype, namely, G protein-coupled inward rectifying current potassium channel type 2 (GIRK2) (15) and forkhead box protein A2 (FOXA2) (28). The effect of transplantation was evaluated for up to 9 months based on motor behaviors of affected hand movements; PET scans using ${ }^{11} \mathrm{C}-\mathrm{CFT}$, which specifically labels DAT; and postmortem histology. Tumorigenicity was also estimated from the results of blood tests and PET scans. The preclinical data obtained thus may extend the applicability of the current autologous cell system as a therapy for PD.

\section{Results}

Evaluation of MSC-DP cells. Cynomolgus monkey MSCs drastically changed their morphology following induction, as reported previously (21): naive MSCs initially showed fibroblast-like mesenchymal cell features (Figure 1A), while induced cells showed a neuron-like morphology with neurite-like processes (Figure 1B). By immunohistochemistry, naive MSCs were negative for neuronal markers, as reported previously (21), but induced cells were positive for neuronal markers, Tuj1 (Figure 1C) and MAP-2 (Figure 1D); dopaminergic neuron markers, TH and DAT (Figure 1, $\mathrm{E}$ and $\mathrm{F}$ ); the A9 dopaminergic neuron marker, GIRK2 (Figure 1G); and a marker of floor plate-derived cells, FOXA2 (Figure $1 \mathrm{H})$. We also confirmed that these cells were positive for sodium channels (Figure 1I), a marker of differentiated neurons.

To confirm whether these cells have an ability to produce and release dopamine, we measured the secretion of dopamine by
HPLC. The amount of dopamine in the culture supernatant was measured following application of high $\mathrm{K}^{+}$depolarizing stimuli, which resulted in release of $1.04 \pm 0.4 \mathrm{pM}$ dopamine per $10^{6}$ cells (Table 1); by contrast, naive cynomolgus monkey MSCs showed no detectable dopamine release. These results are consistent with those of our previous studies: the amount of dopamine release was comparable to the amounts in rats $\left(1.1 \mathrm{pM} / 10^{6}\right.$ cells $)(20)$ and monkeys $\left(0.9 \pm 0.2 \mathrm{pM} / 10^{6}\right.$ cells $)(21)$.

We further investigated the expression of markers specific for A9 dopamine neurons using RT-PCR. The MSC-DP cells expressed GIRK2 and FOXA2 mRNA but not CALB1 mRNA (GIRK2+ $/ \mathrm{FOXA2}^{+}$/ $C A L B 1^{-}$) (Figure 1J). When control tissues obtained from a cynomolgus embryo and an adult animal were analyzed, both showed that the substantia nigra pars compacta ( $\mathrm{SNc}$ ) was strongly positive for GIRK2 and FOXA2 and weakly positive for calbindin, while the ventral tegmental area (VTA) was weakly positive for GIRK2 and FOXA2 but strongly positive for calbindin (Figure 1J). This distinct pattern of GIRK2/FOXA2/calbindin expression in the $\mathrm{SNc}$ and VTA is consistent with those reported in other species, including rodents and humans $(15,28,29)$. We also evaluated the induction efficiency of MSC-DP cells by quantitative immunocytochemistry. Fifty to seventy-five percent of MSC-DP cells were positive for TH, DAT, GIRK2, and FOXA2 (Figure 1K). These findings indicated that the current method efficiently produced MSC-DP cells from the MSCs, which had properties similar to those of the A9 dopamine neurons in the model species.

Behavioral analysis of motor symptoms. The clinical rating scores (CRSs) for parkinsonian animals are shown in Figure 2A. The CRS revealed a significant interaction effect between groups and time 


\begin{abstract}
Table 1
Dopamine-producing capacity of MSC-DP cells and the number of engrafted cells
\end{abstract}

$\begin{array}{ccc}\text { Animal ID } & \begin{array}{c}\text { Dopamine release induced } \\ \text { by } \mathbf{K}^{+}(\mathbf{p M} / \mathbf{1 0} \text { cells })\end{array} & \begin{array}{c}\text { No. of engrafted } \\ \text { cells }\left(\times \mathbf{1 0 ^ { 6 }} \text { counts }\right)\end{array} \\ \text { mon0703 } & 2.6 & 20.4 \\ \text { mon0705 } & 0.5 & 9.0 \\ \text { mon0708 } & 0.4 & 12.0 \\ \text { mon0709 } & 0.8 & 12.7 \\ \text { mon0710 } & 0.9 & 18.6\end{array}$

Dopamine release induced by $\mathrm{K}^{+}$was measured by HPLC. Animals were from the MSC-DP-engrafted group.

after transplantation $\left(F\right.$ distribution $\left.\left[F_{4,32}\right]=3.07, P<0.05\right)$. Thus, we further tested for an effect of time, separately in each of the groups in our study (engrafted or sham), by 1-way ANOVA. The CRS in the MSC-DP-engrafted group showed a marginal effect of time $\left(F_{4,16}=2.95, P=0.055\right)$. In a post-hoc comparison of scores at each time point after engraftment with those at baseline, significant improvements in the CRS were observed at 8 months after engraftment (Dunnett's multiple comparison, $P<0.05$ ). This time effect was not observed in the sham group either by 1-way ANOVA or post-hoc analysis.

Hand-reach scores showed similar time courses (Figure 2B). The scores for the affected hand showed significant group and time interaction $\left(F_{4,32}=2.83, P<0.05\right)$. One-way ANOVA for the repeated measures of hand-reach scores for the affected hand of the MSC-DP-engrafted group revealed a significant effect of time $\left(F_{4,16}=5.62, P<0.001\right)$. A post-hoc comparison of scores at each time point after engraftment with those at baseline revealed significant improvements in hand-reach scores at 4 months (Dunnett's multiple comparison, $P<0.05)$ and 8 months $(P<0.01)$ after engraftment. This effect of time was not observed in the sham group. Therefore, the CRSs and hand-reach scores suggested that the engraftment of MSC-DP cells modestly improved motor behaviors in parkinsonian animals.

Despite these improvements in MSC-DP-grafted animals, spontaneous activities of animals were not affected by any of group (sham vs. MSC-DP engrafted), time (before engraftment and 4 months and 8 months after engraftment), or interaction among these variables (2-way ANOVA with repeated measures, Supplemental Figure 1; supplemental material available online with this article; doi:10.1172/JCI62516DS1). Animals in the engrafted group tended to show higher spontaneous activities than sham-operated animals at 4 and 8 months after engraftment; however, no statistically significant effect of group was observed at any time point in post-hoc analysis (Bonferroni corrected, $P>0.05$ ). Within-subject and between-subject data were highly variable, consistent with a previous report in this type of parkinsonian animal model (30). No dyskinesia-like abnormal movements were observed in any MSC-DP cell-engrafted animals during the observation period.

Figure 2
${ }^{11} \mathrm{C}$-CFT PET. Voxel-based analysis of ${ }^{11} \mathrm{C}$-CFT binding potential $\left(\mathrm{BP}_{\mathrm{ND}}\right)$ images disclosed a significant effect of time in a cluster extending into the dorsal posterior putamen in the engrafted group (Figure 3C) (family-wise error rate [FWE] corrected, $P<0.05$; Table 2). The maximum of this cluster was located ( $x=11.5 \mathrm{~mm}$, $y=-8.0 \mathrm{~mm}, z=3.5 \mathrm{~mm}$ ) in a standard macaque brain space of the Montreal Neurological Institute (MNI) (31) and was safely within the area targeted when engrafting MSC-DP cells into the striatum (Figure $3 \mathrm{~A}$ ). Using this cluster as a region of interest (ROI), we obtained $\mathrm{BP}_{\mathrm{ND}}$ values for this ROI across all animals, groups, and time points. The $\mathrm{BP}_{\mathrm{ND}}$ values obtained are presented in Figure $3 \mathrm{D}$. Two-way ANOVA with repeated measures (Figure 3D) revealed significant effects of an interaction between group and time $\left(F_{4,24}=4.3\right.$, $P<0.01)$. Post-hoc analysis showed that the $\mathrm{BP}_{\mathrm{ND}}$ at 7 days after engraftment was higher than that in the sham-operated group (Bonferroni multiple comparison, $T=4.56, P<0.001$ ), as shown in Figure 3D. In particular, animal mon0703, who received the graft with the largest amount of MSC-DP cells (Table 1), showed the highest $\mathrm{BP}_{\mathrm{ND}}(0.59)$ in the engrafted striatum at 7 days after engraftment, followed by animal mon0710, who showed a BP $\mathrm{ND}_{\mathrm{ND}}$ of 0.43 at 7 days after engraftment (Figure 3B).

The time-dependent decline in ${ }^{11} \mathrm{C}$-CFT binding in the engrafted striatum (Figure 3D) allowed us to analyze the kinetics of ${ }^{11} \mathrm{C}$-CFT binding in detail. Because recent studies have suggested that MSCs are susceptible to senescent (12) and apoptotic changes (13), we supposed that this decline was due to degeneration of engrafted MSC-DP cells. ${ }^{11} \mathrm{C}$-CFT binding is known to be correlated with the density of dopamine neurons or terminals rather than any physiological (or functional) variation in dopamine release. The rate of ${ }^{11} \mathrm{C}$-CFT binding reduction, calculated based on the 1-hit model of neurodegeneration (32), was 0.30 months ( $~ 10$ days) as a half-life period (Figure 3E). This rate of reduction was slightly slower than that for engrafted naive rodent MSCs ( $\sim 3$ days; Supplemental Figure 2) based on previously described data (13). We further tested whether this degenerative process would affect all grafted cells. The plateau of the 1-hit model was significantly higher than the baseline (before engraftment) ${ }^{11} \mathrm{CFT}$ binding level (baseline $\mathrm{BP}_{\mathrm{ND}}=0.087 \pm 0.028$ vs. $\mathrm{BP}_{\mathrm{ND}}$ at plateau $=0.17 \pm 0.029$; Welch's corrected $T_{13}=2.04,1$-tailed $\left.P<0.05\right)$, suggesting that a small portion of the grafted MSC-DP cells survive and integrate

Behavioral assessment. (A) CRSs and (B) hand-reach scores were plotted against time for MSC-DP cell-engrafted (MSC-DP) and sham-operated (Sham) groups. ${ }^{\star} P<0.05$ compared with baseline in the MSC-DP-engrafted group, Dunnett's multiple comparison test.

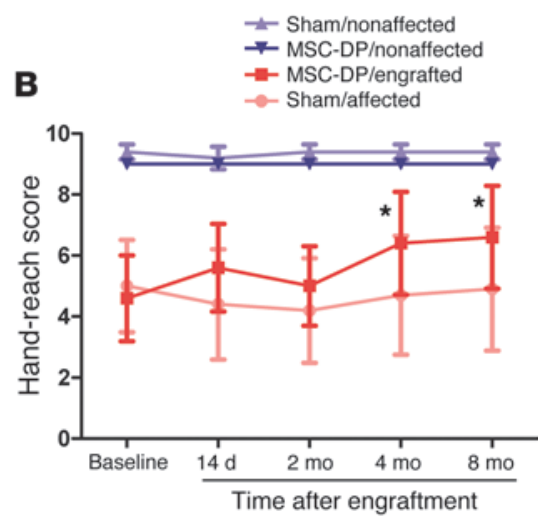

A

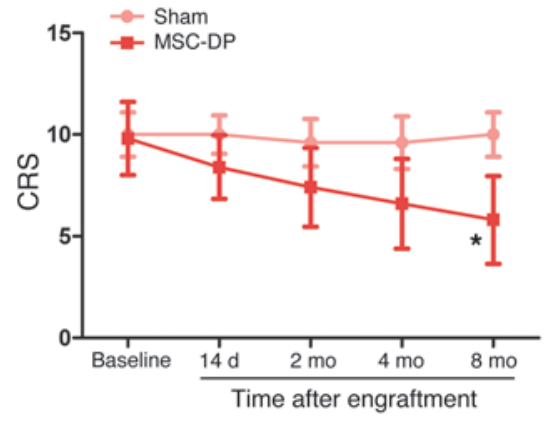


A
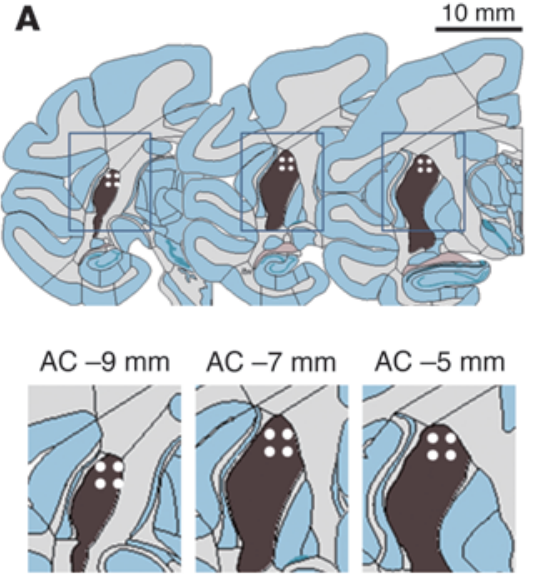

C

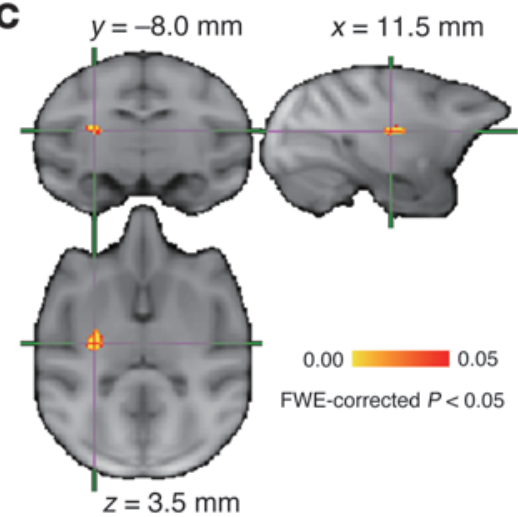

$\mathbf{E}$

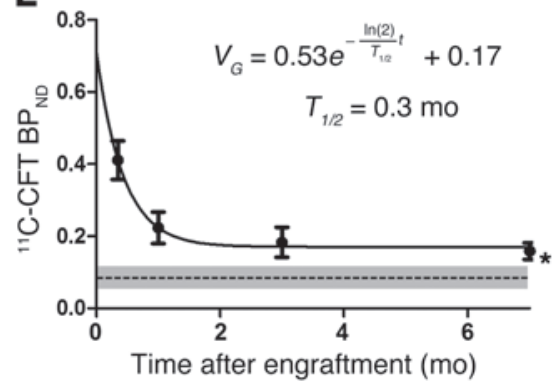

B
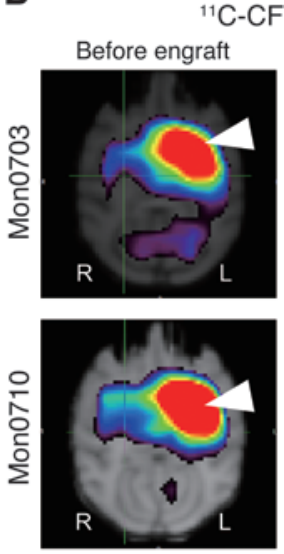

$7 \mathrm{~d}$ after engraft
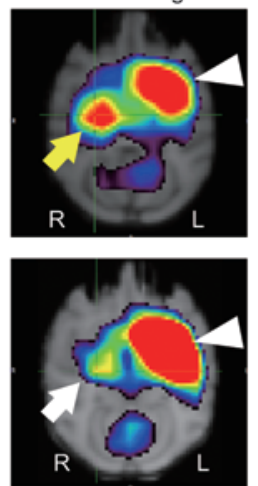

.
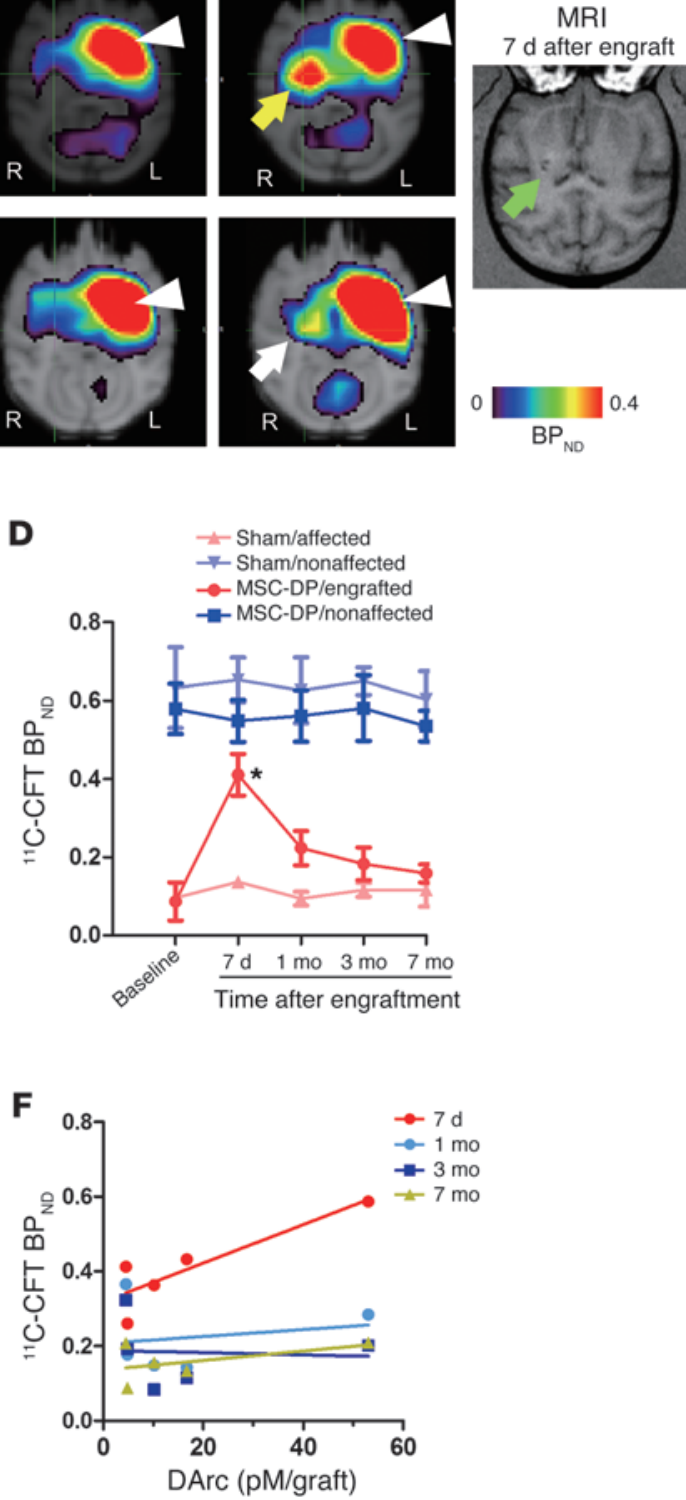

Figure 3

Engrafting and neuroimaging of MSC-DP-engrafted animals. (A) Twelve target points (white dots) for engraftment in putamen in the coronal sections of standard space of cynomolgus macaque. Scale bar: $10 \mathrm{~mm}$. (B) Representative 11C-CFT PET images of MSC-DP-engrafted animals. Seven days after engraftment (engraft), animal mon0703 showed the highest 11C-CFT BP ${ }_{\text {ND }}$ on the MPTP-treated side of striatum (yellow arrow), followed by animal mon0710 (white arrow). Animal mon0710 showed low signal (green arrow) in a T1-weighted MRI after engraftment, which diminished in later scans. Note that the $\mathrm{BP}_{\mathrm{ND}}$ in the non-MPTP-treated (and nonengrafted) side of striatum was very high (white arrowheads) and not different by engraftments. (C) Significant effect of time on 11C-CFT BP ${ }_{\text {ND }}(P$ values range from yellow $[P<0.00]$ to red $[P<0.05])$ overlaid on the study-specific MRI template in MNI space. A cluster with FWE-corrected $P<0.05$ was located in the dorsal posterior putamen in the engrafted striatum. See also Table 2. (D) Time course of $11 \mathrm{C}-\mathrm{CFT} \mathrm{BP}_{\mathrm{ND}}$ in the cluster (in $\mathbf{C}$ ) and contralateral equivalent region. ${ }^{*} P<0.05$ compared with sham, Bonferroni corrected. (E) Time course of 11C-CFT BP ${ }_{N D}$ values in the cluster (in C), fitted by the 1-hit (exponential) model of neurodegeneration. The dashed line and gray area indicate the mean and SEM for the baseline $\mathrm{BP}_{\mathrm{ND}}{ }^{*} P<0.05$ between the baseline $\mathrm{BP}_{\mathrm{ND}}$ and the plateau of the fitted model. $(\mathbf{F})$ The DArc and the $11 \mathrm{C}-\mathrm{CFT} \mathrm{BP}_{\mathrm{ND}}$ values at 7 days and 1,3 , and 7 months after engraftment.

for more than 7 months. This is in contrast to findings in naive MSCs engrafted into rodents, which showed a plateau of the reduction curve returned to the baseline (Supplemental Figure 2), indicating that all naive MSCs may eventually degenerate when transplanted into brain.

We also estimated how the dopamine-releasing capacity (DArc) of grafts related to ${ }^{11} \mathrm{C}-\mathrm{CFT}$ binding after engraftment (Figure 3F). The dopamine-releasing capacity was calculated by multiplication of dopamine release by $\mathrm{K}^{+}$concentration (pM per $10^{6}$ cells) in vitro, as measured by HPLC, and the total number of cells engrafted in each animal $\left(10^{6}\right.$ cells). This graft DArc (pM/ graft) significantly predicted the ${ }^{11} \mathrm{C}$-CFT $\mathrm{BP}_{\mathrm{ND}}$ obtained from the cluster of engrafted striatum scanned at 7 days after engraftment (linear regression, $\mathrm{BP}_{\mathrm{ND}}=5.1 \times 10^{-3}$ $\times \mathrm{DArc}+0.31, F_{1,3}=10.3$, $P<0.05$; Figure 3F) but not those obtained at later time points. Although dopamine release and DAT expression are different measures, both are a common and specific attribute of well-differentiated dopamine neurons (33). Therefore, the findings indicate that the DAT expression at the earliest time point after transplantation is coupled to the dopaminergic capacity of the engrafted cells, while other factors may have degraded this coupling at later time points.

Tests for tumorigenicity and general condition of animals with MSC-DP cell engraftment. There were no abnormal changes in the results of blood tests, including red and white blood cell counts, and blood chemical tests in any of the MSC-DP- and sham-treated animals tested (Supplemental Table 1). There was also no abnormal 
Table 2

Voxel-based statistical results of ${ }^{11} \mathrm{C}$-CFT BP ${ }_{\mathrm{ND}}$ in repeated-measure ANOVA

\begin{tabular}{lccccc} 
Cluster & No. of & \multicolumn{4}{c}{ Maximum voxel } \\
& voxels & $\boldsymbol{x}(\mathbf{m m})$ & $\boldsymbol{y}(\mathbf{m m})$ & $\boldsymbol{z}(\mathbf{m m})$ & F value \\
Right dorsal posterior striatum & 224 & 11.5 & -8.0 & 3.5 & 14.6
\end{tabular}

The location of the maximal voxel is presented in $x, y$, and $z$ coordinates of the standard anterior-posterior commissural coordinates in $\mathrm{MNI}$ space. See Figure $3 \mathrm{C}$ for visual presentation of this cluster. FWE-corrected $P<0.05$.
Immunohistochemical study. Consistent with the ${ }^{8}$ F-FDG PET scans and MRI data, no tumor formation was observed in either group by H\&E staining (data not shown). We further evaluated the proliferative activity of MSC-DP cells engrafted into the striatum by immunostaining using a Ki-67 antibody. Although, we found a small number of $\mathrm{Ki}-67^{+}$cells in the MSC-DP cell-engrafted striatum (Figure 4B), the proportion of $\mathrm{Ki}-67^{+}$cells was $<2.5 \%$, and there was no significant difference between MSC-DP cell-engrafted and control striata (Figure 4C). Moreover, the $\mathrm{Ki}-67^{+}$cells existed

elevation in the levels of the tumor markers carcinoembryonic antigen (CEA), tissue polypeptide antigen (TPA), sialyl Lewis X antigen (SLX), neuron-specific enolase (NSE), and basic fetoprotein (BFP) (Supplemental Table 2).

${ }^{18} \mathrm{~F}-\mathrm{FDG}$ PET scans revealed normal uptake of ${ }^{18} \mathrm{~F}-\mathrm{FDG}$ in the engrafted striatal region of animals in the engrafted group (Figure 4). In MRI scans, 2 animals (mon0710 and mon0049) showed subtle hemorrhagic changes, as suggested by low signals in the engrafted region of the right striatum in T1-weighted MRI images at 7 days after engraftment (Figure 3B); however, no significant signal changes were observed in later MRI scans. Therefore, no enlargement of or tumor formation by the engrafted tissues was suspected, at least at the macroscopic level, up to 8 months after transplantation of MSC-DP cells. These findings were confirmed by histological evaluations, as described below. solitarily, and none of them formed tumor-like masses (Figure 4B).

We then performed triple staining of striatal sections using markers, including those for A9 and A10 dopaminergic neurons. In the transplanted region of MSC-DP cell-engrafted striata, cells positive for DAT and GIRK2 but not for calbindin were clearly observed (Figure 5, A-F), but such cells were not found in any of sham-operated striata. The staining pattern of A9-type dopaminergic neurons was confirmed by analyzing the midbrain of a normal control adult animal with the same triple staining (Supplemental Figure 3): the A9-type dopaminergic neurons in the SNc showed a pattern of $\mathrm{DAPI}^{+} / \mathrm{DAT}^{+} / \mathrm{GIRK}^{+} /$calbindin- $^{-}$(Supplemental Figure $3 \mathrm{~A}$ ), while the A10-type neurons in the VTA had a pattern of $\mathrm{DAPI}^{+} / \mathrm{DAT}^{+} / \mathrm{GIRK}^{-} /$calbindin ${ }^{+}$(Supplemental Figure 3B). We also confirmed that most of the $\mathrm{DAT}^{+}$cells in the engrafted striata were positive for TH (Figure 5, G-J) but not for a glial marker, glial
A

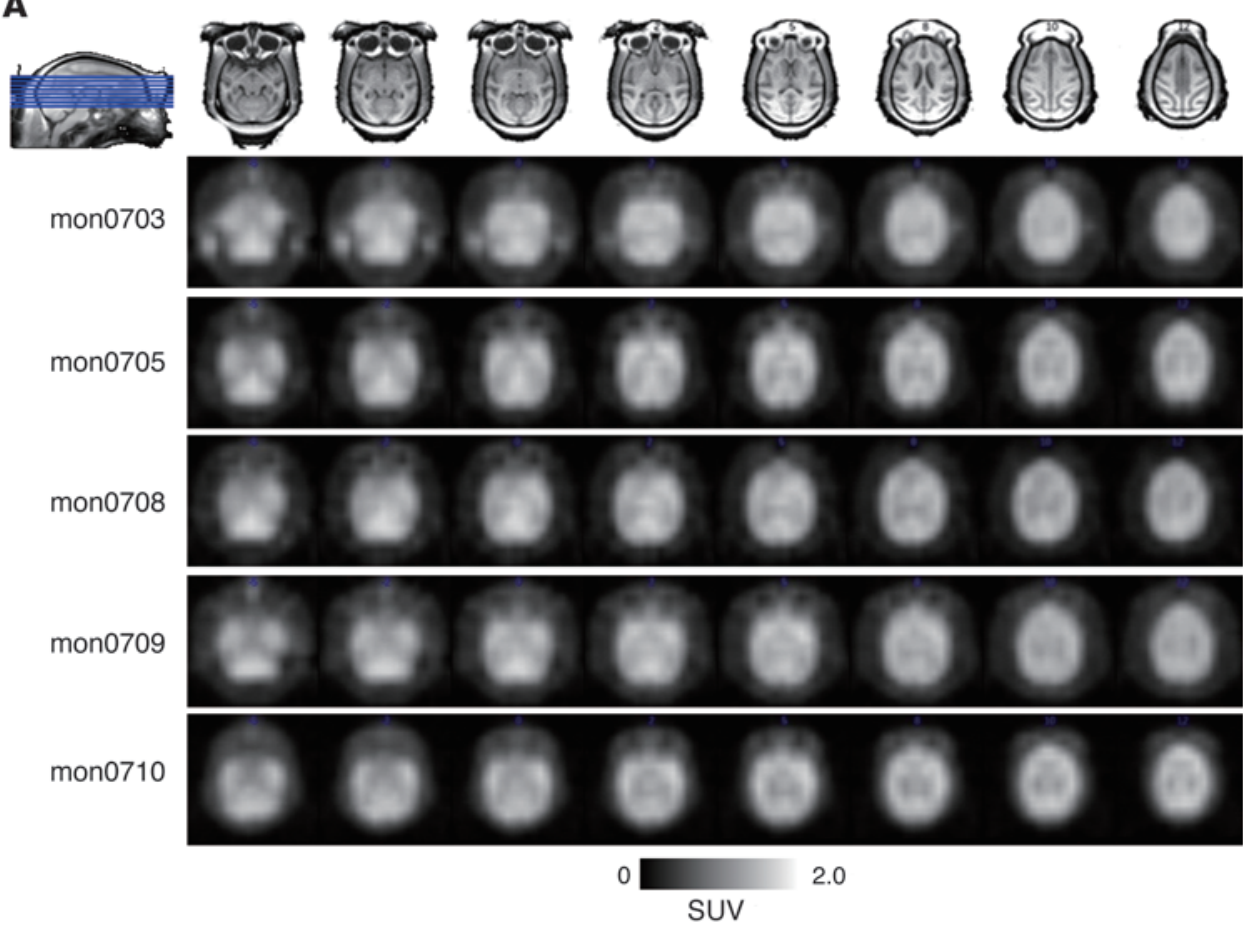

B
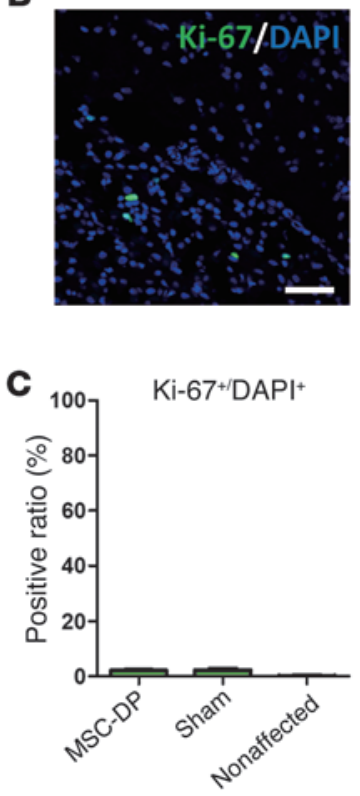

Figure 4

Evaluation of tumorigenicity of MSC-DP-grafted animals. (A) SUV images of ${ }^{18} \mathrm{~F}-\mathrm{FDG}$ PET scans, obtained 8 months after engraftment, are shown for each animal in the engrafted group. No apparently high uptake of ${ }^{18} \mathrm{~F}-\mathrm{FDG}$ was found in any of MSC-DP cell-engrafted animals. (B) Immunostaining with Ki-67 is shown in a section of MSC-DP cell-engrafted striatum. Cells positive for Ki-67 (green) scarcely existed in the dorsal-posterior putamen. Scale bar: $100 \mu \mathrm{m}$. (C) Quantitative analysis of the ratio of Ki-67+ cells to the total number of cells in 3 groups of striatum (MSC-DP grafted, sham operated, nonaffected). See also Supplemental Tables 1 and 2 for the results of blood tests for biochemical and tumor markers. 

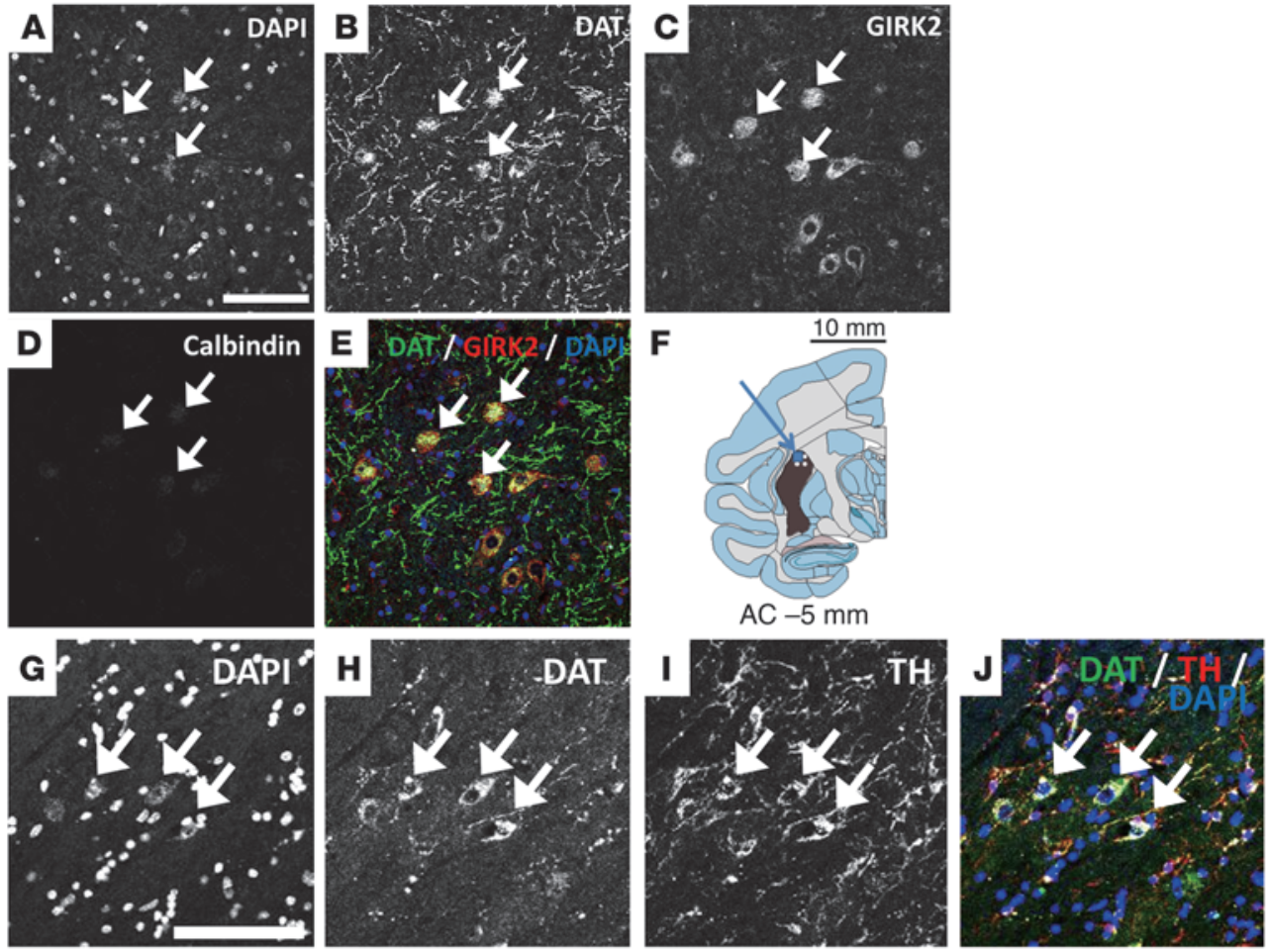

\section{Figure 5}

Immunohistochemistry of the MSC-DP-engrafted striatum. In the MSC-DP-grafted striatum, the cell bodies positive for DAT and GIRK2 (arrows in B and C, respectively) but not for calbindin (arrow in D) were found in the area close to the MSC-DP cellengrafted area (white dots in $\mathbf{F}$ ) in the dorsal posterior putamen. (A) DAPI was used for counterstaining of nuclei. (E) A merged image for DAT, GIRK2, and DAPI is shown in green, red, and blue, respectively. Most of the DAT+ cells were also positive for $\mathrm{TH}$ (arrows in G-J) but not for GFAP (arrows in $\mathbf{K}-\mathbf{N}$ ), while the GFAP+ cells with round-shaped nuclei, putative astroglial cells, were not positive for DAT (arrowheads in $\mathbf{K}-\mathbf{N})$. Scale bar: $100 \mu \mathrm{m}(\mathbf{A}-\mathbf{E}$ and $\mathbf{G}-\mathbf{N}) ; 100 \mathrm{~mm}(\mathbf{F})$.
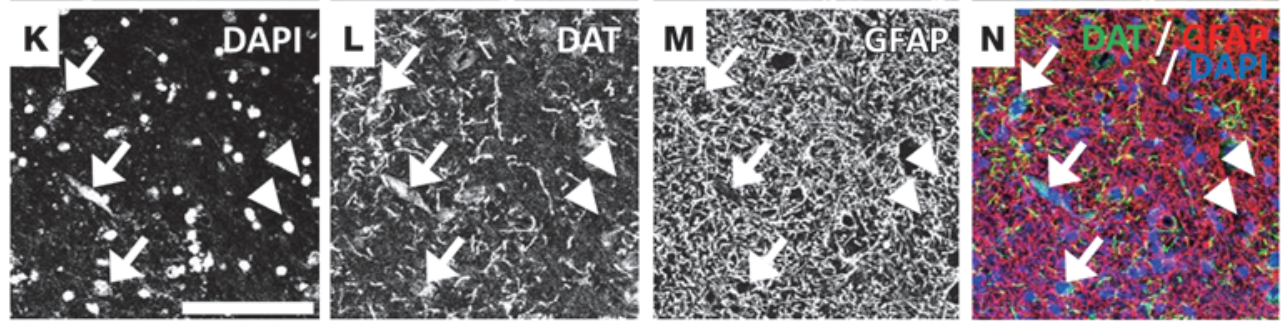

fibrillary acidic protein (GFAP) (Figure 5, K-N). Taken together, these findings indicate that the transplanted MSC-DP cells survived and maintained the characteristics of A9-type dopaminergic neurons for at least 9 months after transplantation.

Quantitative analysis revealed that the number of cells positive for NeuN tended to be higher in the MSC-DP-engrafted striata than in sham and nonaffected (i.e., non-1-methyl-4-phenyl-1,2,3,6-tetrahydoropyridine-treated [non-MPTP-treated]) striata, but no statistically significant differences were found among the 3 groups of striata $\left(F_{3,112}=1.12, P>0.05\right.$; Figure $6, \mathrm{~A}$ and $\mathrm{B}$ ). The number of $\mathrm{TH}^{+}$cell bodies in the engrafted region was higher in the MSC-DP group than in the sham and nonaffected groups, with statistical significance $\left(F_{2,88}=5.99, P<0.005\right.$, Tukey's multiple comparison $P<0.05$; Figure 6 , A and C). In contrast, the number of $\mathrm{TH}^{+}$axon terminals was higher in the MSCDP group than in the sham group, with statistical significance $\left(F_{2,119}=3.93, P<0.05\right.$, Tukey's multiple comparison $P<0.05$; Figure 6, A and D), suggesting that the $\mathrm{TH}^{+}$cell bodies observed in the engrafted striatal group integrated with host striatal neurons by forming new synaptic connections. Similarly, the number of $\mathrm{DAT}^{+}$cells was also higher in the MSC-DP group than in the sham and non-affected striatum groups, with statistical significance $\left(F_{2,74}=16.78, P<0.0001\right.$, Tukey's multiple comparison $P<0.0001$; Figure 6, A and E).

\section{Discussion}

Early transplantation trials for PD treatments used fetal midbrain tissue as a cell source. Successful results were first reported in studies using macaques in the late 1980s (22-24). Subsequent open-label trials in patients with PD also showed that engrafted fetal neurons can survive, appropriately differentiate, and provide striatal dopamine release as measured by PET and showed clinical improvements (34-36), with only one exception (37). Although later double-blind placebo-controlled trials reported no significant clinical benefits $(38,39)$, successful cell replacement therapy in PD would probably be achieved with more sophisticated cell preparation, surgical and patient selection procedures $(2,3)$. In particular, optimization of cell preparation has been an issue when using fetal tissue because of its limited availability and accessibility; thus, there is an urgent need to develop alternative cell sources (40). In addition, recent basic studies based on fetal tissue graft systems have suggested several potential refinements. For example, whether or not the graft contains A9 dopaminergic neurons may be a determinant factor for achieving synaptic formation with host tissues and better behavioral recovery (16). Others have also suggested that expression of DATs in the graft cells alleviates dyskinesias after graft (41). Therefore, proof-of-concept studies using fetal tissue grafts have established the direction of cell-based therapies, including those using stem cells $(2,3,40)$. Particular concerns when 
A
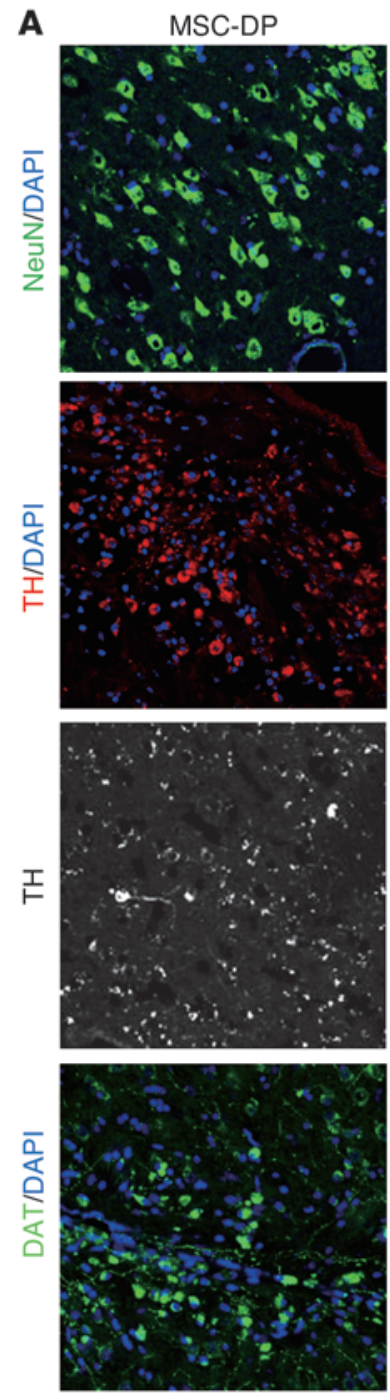
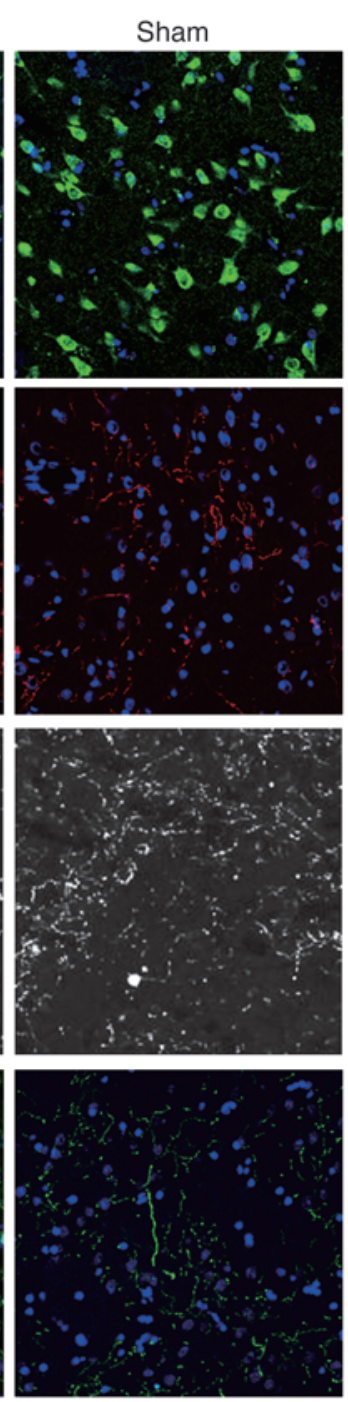

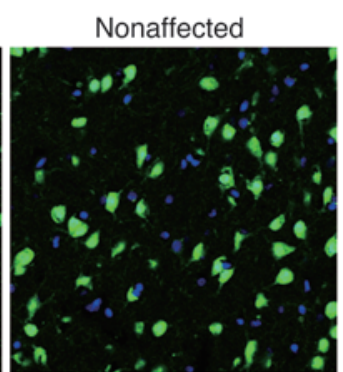

B
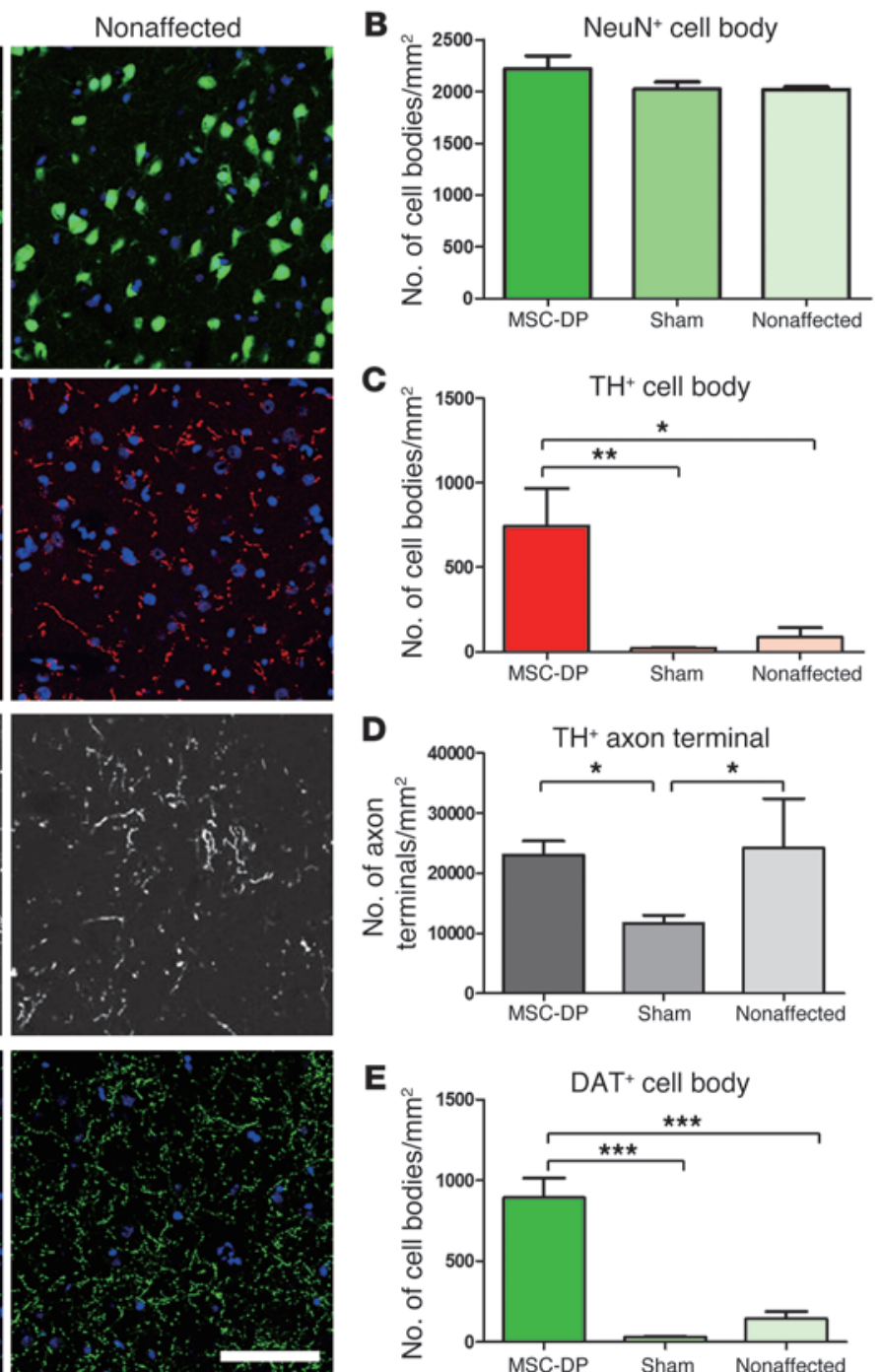

E

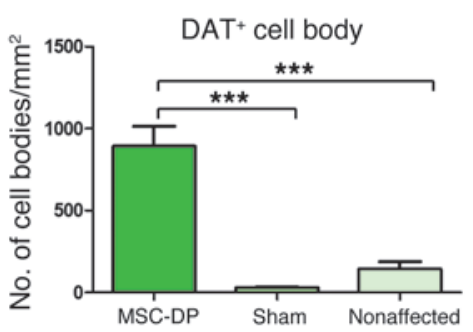

\section{Figure 6}

Quantitative analysis of immunohistochemistry of the dorsal posterior striatum. (A) Immunohistochemical sections of the dorsal posterior striatum for NeuN, $\mathrm{TH}^{+}$cells, $\mathrm{TH}^{+}$terminals, and DAT ${ }^{+}$cells in MSC-DP cell-engrafted animals (MSC-DP), in the sham-operated striatum (Sham), and in the non-MPTP-treated side of the striatum (nonaffected) of sham animals. Scale bar: $100 \mu \mathrm{m}$. (B) Cell counts of $\mathrm{NeuN}^{+}$and $(\mathbf{C}) \mathrm{TH}^{+}$neuronal cell bodies, (D) $\mathrm{TH}^{+}$axon terminals, and (E) DAT+ neurons in the dorsal posterior striatum were plotted for each group. ${ }^{\star} P<0.05$, ${ }^{* \star} P<0.01$, ${ }^{* \star \star} P<0.001$, Bonferroni-corrected multiple comparisons.

applying stem cells are functionality and safety issues after graft, both of which need to be overcome before clinical trials can be commenced (40). From these standpoints, we examined whether the MSC-DP cells could safely function in primate PD model.

Our results can be summarized into the four points. First, the MSC-DP cells induced from macaque MSCs were confirmed to express markers for Tuj1, MAP-2, TH, and DAT and to release dopamine in vitro, as shown previously (21). Moreover, they expressed GIRK2 and FOXA2, specific markers of A9 dopaminergic neurons, but not calbindin, a marker of A10 neurons. Second, when MSC-DP cells were autologously engrafted into the striatum of hemiparkinsonian macaques, motor behaviors, as assessed by CRS and the hand-reach task, gradually improved without accompanying dyskinesia. PET scans detected distinct binding of ${ }^{11} \mathrm{C}$-CFT in the engrafted striatum initially, followed by an exponential decrease in ${ }^{11} \mathrm{C}$-CFT binding. Kinetic analysis suggested that a portion of the MSC-DP graft showed levels of ${ }^{11} \mathrm{C}-\mathrm{CFT}$ binding above the baseline over a period of 7 months. Third, immunohistochemical analysis at 9 months confirmed the increase of $\mathrm{DAT}^{+} / \mathrm{TH}^{+} / \mathrm{GIRK}^{+}$cells and the $\mathrm{TH}^{+}$axon terminals in the engrafted striatum. Fourth, no tumorigenicity was suspected by peripheral blood tests, blood tumor markers, ${ }^{18} \mathrm{~F}$-FDG PET scans, and histology. Therefore, MSC-DP cells are likely to be nontumorigenic and able to remain in the transplanted region until at least 9 months after engraftment, although not in large quantities, thereby modestly restoring dopaminergic function and motor behaviors in vivo.

To the best of our knowledge, this study is the first to show restoration of dopaminergic function and motor behaviors in parkinsonian primate animals following engraftment of MSC-de- 
rived cells. It is notable that kinetic analysis of ${ }^{11} \mathrm{C}$-CFT binding and histology results suggested that at least a portion of MSC-DP cells survived in situ for over 9 months. This is in contrast to naive MSCs, which have much shorter survival times in the brain and disappeared within 3 months in rodent studies; one possible reason for this could be senescent or apoptotic changes $(12,13)$. The long-term survival of MSC-DP cells may have been accompanied by gradual integration into or reinnervation of the host striatal tissues. As shown in Figure 6D, increased numbers of $\mathrm{TH}^{+}$terminals were observed in engrafted animals compared with control animals, suggesting formation of new synaptic connections between the engrafted cells and host tissues; this may at least in part underlie the motor recovery observed at a later time after transplantation of MSC-DP cells. The fact that in vitro DArc was correlated with ${ }^{11} \mathrm{C}$-CFT binding only at early time points after engraftment also supports the hypothesis that other mechanisms, such as integration of MSC-DP cells, into host tissues may be critical for full restoration of motor function.

A notable feature of the current MSC-DP cells is their similarity to A9 dopaminergic neurons. Previously, we reported that dopaminergic neuron-like cells are inducible from MSCs by genetic introduction of NICD, followed by cytokine treatment with bFGF, forskolin, CNTF, and GDNF $(20,21)$. This report further characterized the MSC-DP cells and found that they were double positive for FOXA2 and GIRK2, both specific markers of differentiated A9 dopaminergic neurons. In particular, FOXA2 was recently identified as a transcription factor required for specifying and maintaining the dopaminergic neuron phenotype $(28,42)$ and is considered to be expressed exclusively in floor plate-derived dopaminergic neurons (43). We confirmed the coexpression of FOXA2 and GIRK2 in SNc tissue that contains A9 dopaminergic neurons but not in the VTA, which contains A10 dopaminergic neurons. GIRK2/FOXA2 double-positive cells have not been induced from other stem cell sources using existing protocols (44-46), except for one that manipulates both the sonic hedgehog and WNT signaling pathways $(43,47)$. Recent studies of fetal tissue engrafting in patients with PD or PD model rats have shown that A9 dopaminergic neurons are determinants of successful functional recovery $(16,48)$ and decreased dyskinesia $(49)$ and that they are associated with well-organized synaptic connectivity (16). Overall, the results of our study support a recent hypothesis that well-differentiated stem cells offer benefits for both survival and functional integration with host neural tissues (47).

There are 2 points that favor the suitability of MSC-DP cell transplantation for clinical application. First, the current strategy rests on the autologous cell system. This offers advantages over allograft systems, such as fetal tissue engrafting, in terms of the ethical, social, and political implications. MSCs can be easily collected autologously from the patients' own bone marrow aspirates. Aspiration of bone marrow by itself is widely applied in the field of hematology, and infusion of bone marrow from HLA-matched donors is also commonly performed for treatment of leukemia. Moreover, bone marrow-derived MSC transplantation into brain has been tried clinically without any safety problems (10). The autologous system also has the advantage of not requiring immunosuppression, as compared with allograft systems. Second, no tumorigenicity was suspected up to 9 months after engraftment in primates. Adult stem cells, including MSCs, are known to have less tumorigenic proliferative activity than other cell sources, such as embryonic stem cells. Moreover, the MSC-DP cells expressed
MAP-2, a marker of postmitotic neurons (50), suggesting that they possess minimal risk of tumor formation. A previous study in which MSC-DP cells were engrafted into rodents consistently showed no evidence of tumorigenicity during a 14-week period (20). Schwann cells derived from MSCs also showed no tumorigenicity during a 1-year observation period in our previous study in primates (18). The differentiation technique for converting naive MSCs to MSC-DP cells involved NICD gene introduction by transfection of a plasmid gene but not by retrovirus- or lentivirus-mediated gene transfer, which is known to modify the nuclear genome. The introduced plasmid NICD gene does not appear to have a prolonged effect on the cell cycle, because the introduced NICD gene did not remain in MSCs for a long time (for no longer than 2 passages) (51).

Although our study showed a potential therapeutic effect of MSC-DP cells in a PD model, one may argue that cells pushed to full maturity in vitro would not be the best cell source for grafting (2). For example, grafted dopaminergic neurons obtained from fetal brain are known to survive poorly if their maturation has passed a narrow optimal time window at the time of harvesting (52). Such an argument might be applicable to our results in that the rapid loss of ${ }^{11} \mathrm{C}-\mathrm{CFT}$ binding over time may have been a consequence of the use of differentiated MSCs, namely MSC-DP cells. However, this is still an open question, particularly with respect to stem cells; the relationship between in vitro maturity and survival after graft is not simple and may also depend on other factors, such as the types of cell sources and in vitro preparation. ES grafts with very immature cells seem to cause tumorigenicity, while relatively immature cells, such as neural precursors/neuroblasts, suffer from poor cell survival (40). But more highly differentiated DA neurons recently engineered from ES cells showed good survival (47). Undifferentiated MSCs are known to die very rapidly, within several days after being engrafted into the brain, and this seems to be due to facilitated senescence (12) or programmed cell death of the MSCs (13). Therefore, we have followed the working hypothesis, testing well-differentiated cells derived from MSCs. To address the issue of optimal maturity, cell marker-based subtyping should also be standardized in future studies (33).

A few issues suggest the need for some caution when interpreting our results. First, in this study, we did not label the MSC-DP cells by tagging them with, for example, green fluorescent protein in order to trace the cells after transplantation. This was because we avoided using any extrinsic factors that may pose a safety concern or affect cell survival. As a result, the dopaminergic neuronlike cells found in the striatum may not truly have originated from the grafted MSC-DP cells. However, this is unlikely because the striatum in primates has only a few dopaminergic neurons under normal circumstances: $\mathrm{TH}^{+}$cells number $<1 \%$ of striatal neurons (53) and are preferentially scattered near the white matter-ventral striatum border. In our case, the $\mathrm{TH}^{+} / \mathrm{DAT}^{+} / \mathrm{GIRK}^{+}$cells were located only in an area near the engrafted area in the dorsal striatum (Figure 5), and the number of the cells was larger than that in sham and nonaffected striata (Figure 6, C and E). Second, regarding PET ligands, a well-known ligand for DA, ${ }^{18} \mathrm{~F}-\mathrm{DOPA}$ was not used in this study, because it is a metabolic ligand for amino acids and may reflect not only dopamine turnover in dopaminergic neurons, but also uptake of amino acid precursors in tumors (54) or inflammation (55). Therefore, ${ }^{11} \mathrm{C}$-CFT was carefully chosen as an in vivo surrogate marker for tracking MSC-DP cells, because it is a highly specific ligand for DAT, which is specif- 
ically expressed in dopaminergic neurons and was confirmed to be present in the current MSC-DP cells in vitro. The high uptake of ${ }^{11} \mathrm{C}$-CFT was not found in any of the sham-operated animals, even though they received PBS injections, which may induce tissue damage or inflammation. Third, the study does not clarify the complete mechanism underlying the motor recovery in the current animals. PET findings revealed increased DAT expression only in the early phase, while behavioral data showed significant recovery only in the later phase. As described above, we believe the discrepancy is explained by a time delay required for the remaining MCS-DP cells to achieve synaptic connections with the host tissues. This speculation should be clarified in the future study, since the delayed motor recovery found here seems to be common in trials of cell replacement therapies in PD. For example, fetal tissue grafting improved motor symptoms at 6 to 9 months after grafting in selected patients with PD (39). ES cell-derived well-differentiated DA neurons also restored motor behaviors 3-5 months after grafting in an animal model (47). Finally, these data do not show convincing evidence that benefits were truly results of an in vitro process differentiating MSCs into A9 DA neurons. We cannot completely exclude the possibility that undifferentiated MSCs, contaminated in the graft and not positive for A9 markers $(25 \%-50 \%$ of cells; Figure $1 \mathrm{~K})$, might have contributed to the observed benefits, for example, by way of trophic effects. Therefore, future studies should directly compare the MSC-DP cell with naive MSC transplantation.

In conclusion, this study indicates that the autologous MSC-differentiation system may be safe and potentially effective for restoring motor dysfunction in hemiparkinsonian nonhuman primate animals. Although further studies are needed to improve the viability of MSC-DP cells and net performance for functional recovery, the current system may expand the availability of cell sources for cell-based therapies for patients with PD.

\section{Methods}

Ten adult male cynomolgus monkeys (Macaca fascicularis; body weight, 3-4 $\mathrm{kg}$ ) were used in this study. All animals were housed in a room kept at $26^{\circ} \mathrm{C}$. Animals were pretreated with the neurotoxin MPTP to generate a hemiparkinsonian model, and their bone marrow aspirates were used for induction of autologous MSC-DP cells. Five animals were used for the engraftment of MSC-PD cells ( $n=5$, MSC-DP group), and the remaining 5 underwent a sham operation in which they received injection of PBS only ( $n=5$, sham group).

Isolation of MSCs from bone marrow aspirates. Primary MSCs were isolated from bone marrow aspirates obtained from the iliac bone of every animal in the engrafted group. Bone marrow was aspirated from each animal 3-4 months prior to transplantation under deep anesthesia achieved by intramuscular injection of ketamine $(6.6 \mathrm{mg} / \mathrm{kg})$, xylazine $(1.3 \mathrm{mg} / \mathrm{kg})$, and atropine $(0.01 \mathrm{mg} / \mathrm{kg})$. For aspiration, we used a bone marrow needle (18 G; Sheen-man Co. Ltd.) and 2.5-ml aspiration syringes containing 100 units of heparin (Novo-Heparin, Mochida Pharmaceutical Co. Ltd.) to prevent clotting. Aspirates were diluted 1:10 using culture medium $(\alpha$-minimum essential medium [ $\alpha$-MEM] plus $15 \%$ FBS plus $2 \mathrm{mM}$ L-glutamine plus kanamycin) and incubated at $37^{\circ} \mathrm{C}$ in $5 \% \mathrm{CO}_{2}$. After 4 days, nonadherent cells were removed by replacing the medium. Adherent cells, namely, MSCs, were subjected to subculture when they reached $95 \%$ confluence and were subcultured 4 times. Finally, they were subjected to dopaminergic neuronal differentiation.

Differentiation of MSC-DP cells from MSCs. MSC-DP cells were differentiated from MSCs using a method of spermine-pullulan-mediated reverse transfection, as described previously (21). We used pullulan, with an average molecular weight of 47,300 Da (Hayashibara Biochemical Laboratories) and spermine (Sigma-Aldrich). A cationized pullulan derivative was prepared as described previously (21), and a gene encoding mouse NICD (constitutively active form), including a transmembrane region with a small fragment of extracellular domain and the entire intracellular domain, was subcloned into the pCI-neo vector (Promega) (pCI-NICD). Cells were further selected by G418 for 3 to 4 days, replated at a cell density of 2,080 cells per $\mathrm{cm}^{2}$, and treated with $5 \mu \mathrm{M}$ forskolin (Calbiochem), $10 \mathrm{ng} / \mathrm{ml} \mathrm{bFGF}$ (Peprotech), and $10 \mathrm{ng} / \mathrm{ml} \mathrm{CNTF} \mathrm{(R \& D} \mathrm{Systems)} \mathrm{in}$ $\alpha$-MEM containing 5\% FBS for 5 days. Finally, GDNF (50 ng/ml; Peprotech) was added.

Evaluation of MSC-DP cells - dopamine release assay using HPLC. The dopamine release assay was performed as described previously (20,21). Briefly, cells were washed in a low $\mathrm{K}^{+}$solution for 5 minutes, and the medium was replaced with a high $\mathrm{K}^{+}$solution for 5 minutes. The concentration of released dopamine was determined using HPLC with a reverse-phase column and an electrochemical detector system (Eicom Corporation). The mobile phase was composed of $0.1 \mathrm{M}$ phosphate buffer ( $\mathrm{pH}$ 6.0), $20 \%$ methanol. The amount of dopamine release was measured according to the number of cells, which were counted after trypsinization.

Evaluation of MSC-DP cells - immunocytochemistry. The primary antibodies used were anti-MAP-2ab (1:250; Sigma-Aldrich) and anti-Tuj1 (1:100; $\mathrm{BAbCO})$ as neuron-specific markers and anti-TH (1:1,000; Chemicon, Millipore), anti-DAT (1:100; Millipore), anti-GIRK2 (1:80; Alomone Labs), and anti-FOXA2 (1:50; Santa Cruz Biotechnology Inc.) as markers of dopaminergic neurons. We also used an anti-sodium channel antibody (PAN) (clone K58/35, 1:1,000; Sigma-Aldrich) as a marker of neurons. Secondary antibodies were anti-mouse or anti-rabbit IgG antibodies conjugated to Alexa Fluor 488 (Invitrogen) and anti-rabbit or anti-goat IgG conjugated to Cy3 (Jackson ImmunoResearch Laboratory). Nuclei were counterstained with DAPI and inspected using confocal laser microscopy (Nikon Corporation).

Evaluation of MSC-DP cells - RT-PCR. We analyzed the relative expression levels of GIRK2, FOXA2, and CALB1 mRNAs using RT-PCR to classify MSC-DP cells as A9-type neurons (GIRK2 $\left.2^{+} / \mathrm{FOXA2}^{+} / \mathrm{CALB1} 1^{-}\right)$or A10-type neurons $\left(\right.$ GIRK2-/FOXA2-/CALB1 $\left.1^{+}\right)(28,29,56)$. The amount of cDNA was normalized on the basis of the signal from the ubiquitously expressed $\beta$-actin. The primers were designed based on the cDNA sequence of the Macaca mulatta, which is highly homologous to that of Rattus norvegicus, as revealed by an alignment search tool (BLAST; http://www.ncbi.nlm.nih. gov/BLAST/). The primer sequences and precise conditions were as follows: $\beta$-actin, $5^{\prime}$-TCTAGGCGGACTGTGACTTACTTGCGTTAC-3' (forward) and 5'-AATCAAAGTCCTCGGCCACATTGTAGAACT-3' (reverse) (TM, melting temperature; $60^{\circ} \mathrm{C}, 25$ cycles); GIRK2, 5'-ATCCAGAGGTATGTGAGGAAAGATG-3' (forward) and 5'-CACTGTGTAAACCATGACGAAAATC-3' (reverse) (TM, melting temperature; $55^{\circ} \mathrm{C}, 40$ cycles); calbindin, 5'-GCTGTATGATCAGGACGGCAAT- $3^{\prime}$ (forward) and 5'-TCTAGTTATCCCCAGCACAGAGAA-3' (reverse) (TM, melting temperature; $58^{\circ} \mathrm{C}, 40$ cycles); and FOXA2, 5'-CGGTGTTGCAGAGACGAAAG-3' (forward) and $5^{\prime}$-CAGAATCTGCAGGTGCTTGAAG-3' (reverse) (TM, melting temperature; $58^{\circ} \mathrm{C}, 40$ cycles). As a positive control for GIRK2, FOXA2, and calbindin, total RNA was collected and analyzed using 2 types of brain tissues from cynomolgus embryos ( 6th fetal month, Macaca fascicularis) and an adult animal (8 years old, Macaca fascicularis), namely, the SNc and VTA.

MPTP-induced hemiparkinsonism. The animal model of the unilateral PD was produced using the neurotoxin MPTP, as previously reported $(30,57)$, with some modifications. Briefly, animals received slow arterial injections of MPTP into the right carotid artery. Unlike previous studies that accessed the carotid artery by incision of the upper cervical area, we 
applied an angiographic approach that allowed us to access the target artery less invasively and did not require postoperative occlusion of the target artery, which causes insufficient cerebral circulation. We used an 80-cm-long catheter (3F, CATEX Co. Ltd.) and X-ray angiographic device (OEC 8800, GE Healthcare) to place the catheter into the internal carotid artery. After the tip of the catheter was placed in the proximal (cervical) portion of the internal carotid artery, the MPTP solution $(0.4 \mathrm{mg} / \mathrm{kg}$ dissolved in $40 \mathrm{ml}$ of saline, $\mathrm{pH} 7.4$ ) was infused at a rate of $2 \mathrm{ml}$ per minutes. In every animal, the right pupil showed dilatation with a diameter $>5$ $\mathrm{mm}$, indicating the adrenergic effect of MPTP (58). All animals recovered safely from anesthesia and exhibited hemiparkinsonism with a crooked arm posture and dragging leg, with slow or disabled movements in the left hand and arm. All animals continuously revealed hemiparkinsonism over a period of 3 months, and none of them showed spontaneous recovery from motor disabilities.

Engrafting MSC-DP cells into the striatum. Under deep anesthesia, each animal in the engraftment group underwent MRI-guided stereotactic surgery for engrafting of MSC-DP cells into the putamen on the right side (the same side as the MPTP infusion). The animal's head was fixed to a stereotactic acrylic holder in a sphinx position, the head skin and muscles were incised under aseptic conditions after subcutaneous injection of lidocaine (Xylocain 2\%, AstraZeneca PLC), and the surface of the cranium bone was exposed by separating the galea aponeurotica. Three to four MRI-visible markers were attached to the fringe of the exposed cranium to be used as a reference for co-registering the translocation between MRI and the stereotactic micromanipulator. Then, the stereotactic holder was placed horizontally on the MRI gantry with its axis parallel to the $z$ axis of the MRI bore, and a high-resolution head MRI scan was obtained with the inversion recovery-fast spoiled gradient recalled echo (IR-FSPGR) sequence. Obtained MRI images were transformed with a 3D translocation algorithm to the standard macaque brain template (31) and resliced in a volume with a matrix of $110,134,80$ voxels, a cubic voxel size of $0.5-\mathrm{mm}$ edge length, and an origin at the midportion of the anterior commissure (AC). On 3 coronal views of the MRI image sectioned at AC $-5,-7$, and $-9 \mathrm{~mm}$, we determined 12 target points for transplantation (Figure $3 \mathrm{~A}$ ). For each of the 12 targets, the relative coordinates were read on the MRI image in reference to one of the markers on the cranium. After MRI scans were finished, the head holder was then attached to a customized metallic stereotaxic apparatus on which the stereotactic coordinates of the markers were read using a micromanipulator (SM-15 Narishige Group). Then the target coordinates in the micromanipulator were determined based on the coordinates of the reference markers in the MRI image and their location relative to that of the micromanipulator.

Immediately before injecting cells, the autologous MSC-DP cells were collected and suspended in $60 \mu \mathrm{l}$ of $0.1 \mathrm{M} \mathrm{PBS}$, and the number of cells was counted. The average total cell count was $14.0 \times 10^{6} \pm 5.0 \times 10^{6}($ mean $\pm \mathrm{SD})$, with a range of $9.0 \times 10^{6}$ to $20.4 \times 10^{6}$ (Table 1 ). For each target coordinate of $x$ and $y$ determined by above method, the tip of a 10- $\mu 1$ Hamilton syringe with a 30-gauge needle was slowly advanced vertically and kept in place at the target coordinate for $z$. Then MSC-DP cells in PBS solution were injected at a rate of $0.5 \mu \mathrm{l}$ per minute until a total of $5 \mu \mathrm{l}$ had been injected at each target site. The needle was kept in place for an additional 3 minutes to allow the injectate to diffuse and then slowly retracted to minimize diffusion of the injectate over the cortical surface. The sham operation group received injection of the same amount of PBS solution in the same 12 target points in the right dorsal posterior putamen.

Behavioral analysis of motor function. Behavioral performance was assessed using the CRSs, hand-reach scores for the affected hands, and spontaneous activities in housing cages. The previously validated CRSs were used to quantitatively assess the parkinsonian status of the monkeys $(59,60)$. The scale consists of ratings of posture ( 0 to 2 ), gait ( 0 to 5 ), bradykinesia (0 to 5), balance (0 to 2 ), tremor for each arm (0 to 3), gross motor skills ( 0 to 4 for each arm), defense reaction ( 0 to 2 ), and freezing ( 0 to 2 ). The score was the sum of the features out of a total of 32 points, with 0 corresponding to normal and 32 corresponding to severe disability. Scores were assessed before and 14 days, 2 months, 4 months, and 8 months after engrafting and were evaluated by an experimenter blinded to the group allocation.

Hand-reach scores were assessed based on food-taking behavior by the affected hand, as described previously $(30,61)$, and were assessed at the same time points as CRSs (before and 14 days, 2 months, 4 months, and 8 months after engraftment). Five $\sim 5-\mathrm{mm}^{3}$ pieces of apple were placed in front of a circular hole (6-cm diameter) in the lower corner of the cage, so that the animals could pick up the pieces and eat them. Five trials were performed for each session, and three sessions were performed on each of three consecutive days at each time point. Evaluations were made before engrafting and 14 days, 2 months, 4 months, and 8 months after engrafting. All behaviors during the task were monitored and recorded by video. After collection of all data, an experimenter blinded to the group allocation of animals scored the movement velocity of the proximal forelimb (arm and forearm) on a scale of 0 to 5 ( 0 means very fast, 5 means very slow), the movement velocity of hand grasping and finger pinching on a scale of 0 to 4 , and the accuracy of food picking on a scale of 0 to 2 . The sum of these items was recorded as the hand-reach score, with 11 corresponding to normal and 0 corresponding to severe disability.

We also measured spontaneous body activities by monitoring movements using a passive infrared system for monitoring spontaneous animal activities (Supermex, Muromachi Kikai Co. Ltd.) before and 14 days, 4 months and 8 months after engraftment. The animals were placed in a 600 -mm-wide $\times 700$-mm-deep $\times 740$-mm-high cage, in which they were housed for acclimation beforehand. Activities were monitored consecutively for a period of 3 days ( 72 hours). The infrared sensor was attached so as to cover the whole cage in a single field of view. Data were imported to a computer and analyzed using a dedicated program for counting the number of pixels showing signal changes in a 10-minute time frame.

PET. We performed ${ }^{11} \mathrm{C}$-CFT PET scanning to evaluate DAT expression in cynomolgus brains, as described previously $(26,62)$. The tracer ${ }^{11} \mathrm{C}-\mathrm{CFT}$ was prepared by a conventional method using ${ }^{11} \mathrm{C}$-methyl-triflate (63) and a precursor, nor $\left[\right.$ methyl- $\left.{ }^{11} \mathrm{C}\right] 2-\beta$-carbomethoxy- $3-\beta$-(4-fluorophenyl)tropane. The animals received intramuscular injections of ketamine and xyladine, followed by continuous intravenous infusion of propofol $(6 \mathrm{mg} / \mathrm{kg} / \mathrm{h})$ to achieve anesthesia, and their respiration was assisted using an anesthetic machine (Cato, Drager) with an inspired gas mixture of $\mathrm{O}_{2}$ and $\mathrm{N}_{2}\left(\mathrm{O}_{2} / \mathrm{N}_{2}=30 \%: 70 \%\right)$. We monitored endotidal $\mathrm{CO}_{2}$ level $\left(\mathrm{EtCO}_{2}\right)$ and percutaneous oxygen saturation level $\left(\mathrm{SpO}_{2}\right)$, and, if needed, adjusted the respiration rate to achieve $\mathrm{SpO}_{2}>95 \%$ and $\mathrm{EtCO}_{2}$ of $35 \mathrm{mmHg}$. After achieving deep anesthesia, animals were placed on the PET gantry, and their bodies were kept warm by using a temperature managing unit (Bair Hugger Model 505, Arizant Inc.). A 15-minute transmission scan was obtained using a $68 \mathrm{Ge} / 68 \mathrm{Ga}$ rod source for attenuation correction; then, $187 \mathrm{MBq}$ of $\left[{ }^{11} \mathrm{C}\right]-\mathrm{CFT}$ was slowly injected intravenously over a period of 1 minute, before which a 2D-dynamic PET scan lasting for 60 minutes was commenced, with scan data obtained by 39 time frames ( 18 frames of $10 \mathrm{~s}, 6$ frames of $30 \mathrm{~s}, 7$ frames of $120 \mathrm{~s}$, and 8 frames of $300 \mathrm{~s})$. PET scans were performed on a PET scanner (ECAT ACCEL, Siemens Healthcare) that provided 47 slices in an axial field of view of $162 \mathrm{~mm}$ with an intrinsic transaxial resolution of $6.2 \mathrm{~mm}$ in full width at half maximum (64). The PET emission data were corrected for attenuation using the transmission data, and images were reconstructed in a matrix of $128,128,47(x, y, z)$ with a voxel size of $0.86,0.86,3.38 \mathrm{~mm}(x, y, z)$ using a filtered back projection algorithm with a $2-\mathrm{mm}$ Gaussian filter. 
DAT binding in the brain was quantified voxel by voxel as a $\mathrm{BP}_{\mathrm{ND}}$ image based on a simplified reference tissue model (65). The cerebellum was considered as a reference region and was identified by manual delineation of its boundary on a T1-weighted image that was pre-realigned to the time-integrated PET image. We also generated a tracer delivery image (R1) to be used for the next step of co-registration between the PET and MRI images. Calculation of $\mathrm{BP}_{\mathrm{ND}}$ and $\mathrm{R} 1$ was performed using an open-source program (PyBLD; http://www.mi.med.osaka-u.ac.jp/pybld/pybld.html).

Tests for tumorigenicity and general conditions of engrafted animals. To assess the safety of our engrafting procedure, we performed blood tests, including red blood cell counts, white cell counts, and blood chemical tests, in all test animals to evaluate general conditions. We also analyzed the blood for several tumor markers among those tested in the clinic (66), including CEA, TPA, SLX, NSE, and BFP.

We also performed ${ }^{18} \mathrm{~F}$-FDG PET to assess any tumorigenic changes in the grafted area using a previously described method (18). After induction of anesthesia, we injected $185 \mathrm{MBq}$ of ${ }^{18} \mathrm{~F}-\mathrm{FDG}$ and scanned the head region for a period from 40 to 60 minutes after injection. ${ }^{18} \mathrm{~F}-\mathrm{FDG}$ scans were performed in a $3 \mathrm{D}$ mode after the transmission scan. The reconstructed ${ }^{18} \mathrm{~F}$-FDG radioactivity image (in $\mathrm{Bq} / \mathrm{ml}$ ) was scaled based on the injected dose of ${ }^{18} \mathrm{~F}-\mathrm{FDG}$ per body weight of animal (Bq/g) to calculate images of standardized uptake value (SUV). The region was considered to be abnormal when the SUV was greater than 2.5 (67).

MRI. MRI was performed to enable MRI-guided stereotaxic surgery and evaluation of graft size after transplantation and for the analysis of PET data, using a 3-Tesla MRI scanner (Signa LX VAH/I, GE Healthcare). We obtained 3D T1-weighted MRI images from each animal with an IR-FSPGR sequence with imaging parameters as follows: $\mathrm{TR}=9.4 \mathrm{~ms}, \mathrm{TE}=2.1 \mathrm{~ms}$, $\mathrm{TI}=600 \mathrm{~ms}$, matrix $=128 \times 128$, field of view $=99.8 \mathrm{~mm}$, slice thickness $=$ $0.7 \mathrm{~mm}$, number of slices $=100$.

Immunohistochemical analysis of engrafted striatum. Nine months after transplantation, animals were sacrificed by overdose of pentobarbital and perfused transcardially with $4 \%$ paraformaldehyde in $0.1 \mathrm{M}$ PBS. Brain tissues were cut into $10-\mu \mathrm{m}$-thick frozen coronal sections at the level of the posterior striatum (from $y=-7$ to $-9 \mathrm{~mm}$ ) using a cryostat. Primary antibodies used for immunohistochemistry were as follows: NeuN (1:150; Millipore), TH (1:1,200; DAKO), DAT (1:1,000; Millipore), GIRK2 (1:1,000; Chemicon, Millipore), calbindin (1:50; Swant), and Ki-67 (1:400; Thermo Scientific). Secondary antibodies were anti-rabbit IgG conjugated to Alexa Fluor 488 (Invitrogen), anti-rat IgG conjugated to Alexa Fluor 488 (Invitrogen), or anti-rabbit and anti-rat IgG conjugated to Cy3 (Jackson ImmunoResearch Laboratory). For triple staining for DAT/GIRK2/ calbindin, calbindin was visualized using a anti-mouse IgG antibody conjugated to Alexa Fluor 680 (Invitrogen). Costaining with DAPI was also performed to confirm nuclear staining. For quantitative analysis, we chose 3 coronal sections, approximately located at $y=-5,-7,-9 \mathrm{~mm}$, and randomly selected 79-124 fields of view with an area of $0.316 \times 0.316$ $\mathrm{mm}^{2}$ within the dorsal posterior putamen, and counted the number of cells stained for NeuN, TH, or DAT. The counts were compared among sections of striatum that received engraftments (MSC-DP group), those of sham-operated striatum (sham group), and those from the contralateral side of the striatum (non-MPTP-treated group) of sham-operated animals (nonaffected group). For counting $\mathrm{TH}^{+}$terminal density, images captured under a $\times 20$ objective lens were imported into ImageJ (http:// rsbweb.nih.gov/ij/); thereby, color was decomposed into red, green, and blue and thresholded for red based on the automatic isodata algorithm. To estimate the number of $\mathrm{TH}^{+}$terminals, the numbers of red particles, with sizes of $>4$ and $<2,000$ pixels (corresponding to $>4 \mu \mathrm{m}^{2}$ and $<4 \times 10^{6}$ $\mu \mathrm{m}^{2}$ ), were counted. The data were expressed as the number of cells or axonal terminals per area $\left(\mathrm{mm}^{2}\right)$.
Statistics. Values are expressed as mean \pm SEM. For immunohistochemistry and behavioral tests, data were analyzed using ANOVA. Differences among means were further analyzed by post-hoc multiple comparisons. The threshold for statistical significance was $P<0.05$. Statistical analysis was performed using PRISM software (GraphPad Software Inc.).

For ${ }^{11} \mathrm{C}$-CFT PET images, $\mathrm{BP}_{\mathrm{ND}}$ images were preprocessed for voxel-based statistics using a previously described method (62). Briefly, the PET images were co-registered with MRI images (obtained at the time closest to the PET scan) using a rigid body transformation and transformed to the standard brain space of cynomolgus macaque (31). The transformation matrix of MRI images to the standard space was determined by an affine transformation algorithm. Voxel-based statistics were performed using a nonparametric permutation test (68) with 1-way ANOVA with repeated measures of $\mathrm{BP}_{\mathrm{ND}}$ in the engrafted group. Permutation was performed within data from each subject, respecting the repeated-measures structure of the data. We are interested in the statistical $F$ contrast for the effect of time in which FWE-corrected $P$ values of less than 0.05 were applied using a thresholdfree cluster enhancement (69). The significant cluster thus obtained (see Figure 3C) was used as a ROI for subsequent analyses (Figure 3, D-F). We also obtained $\mathrm{BP}_{\mathrm{ND}}$ values from the contralateral (nonaffected) dorsal putamen as a control for $\mathrm{BP}_{\mathrm{ND}}$ values, by ROI flipping (left-right) of the above cluster. The control values for $\mathrm{BP}_{\mathrm{ND}}$ were $0.56 \pm 0.12$ (95\% confidential interval, $0.51-0.61)$ in the engrafted group and $0.63 \pm 0.11(95 \%$ confidential interval, $0.59-0.67)$ in the sham group. Image analysis was performed using FSL tools (http://www.fmrib.ox.ac.uk/fsl).

The 1-hit model of neurodegeneration with a constant risk (32) was fitted to the time course data using $\mathrm{BP}_{\mathrm{ND}}$ values obtained from the ROI in the engrafted striatum or using the graft volume of naive MSCs obtained from Figure 1 in ref. 13. The actual equation for the model was $V_{G}=V_{0} \times$ $\exp \left(-\ln [2] \times t / T_{1 / 2}\right)+V p$, where the $V_{G}$ is the time course of graft $\mathrm{BP}_{\mathrm{ND}}$ or volume; $V_{0}$ is the initial value for $V_{G}$; $\exp$ is the exponential function; $t$ is the time after grafting; $T_{1 / 2}$ is a half-life period; and $V p$ is plateau. Nonlinear fitting was performed using PRISM software.

Study approval. The experimental protocol and design of the study were in line with the institutional guides for animal experiments and the NIH Guide for the Care and Use of Laboratory Animals (NIH Publication No. 80-23) and were approved by the institutional committee for animal care and experiments of Tohoku University, RIKEN, and the National Cerebral and Cardiovascular Center.

\section{Acknowledgments}

We thank Noboru Teramoto, Hajime Fukuda, Hiroshi Koshino, Kyoko Shioya, Keiko Tokuda, and Akihiro Kawasaki for their technical help and Tsuyoshi Tahara and Hideki Mochizuki for their helpful comments. This study was supported by the Program for Promotion of Fundamental Studies in Health Sciences of the National Institute of Biomedical Innovation (NIBIO, 05-06, 10-05).

Received for publication July 13, 2012, and accepted in revised form September 28, 2012.

Address correspondence to: Takuya Hayashi, Functional Probe Research Laboratory, Center for Molecular Imaging Science, RIKEN, 6-7-3 Minnatojima-minamimachi, Chuo-ku, Kobe, Hyogo 650-0047, Japan. Phone: 81.78.304.7121; Fax: 81.78.304.7123; E-mail: takuya. hayashi@riken.jp. Or to: Mari Dezawa, Department of Stem Cell Biology and Histology and Department of Anatomy and Anthropology, Tohoku University Graduate School of Medicine, 2-1 Seiryo-machi, Aoba-ku, Sendai, Miyagi 980-8575, Japan. Phone: 81.22.717.8025; Fax: 81.22.717.8030; E-mail: mdezawa@med.tohoku.ac.jp. 
1. Gage FH. Cell therapy. Nature. 1998; 392(6679 suppl):18-24.

2. Brundin P, Barker RA, Parmar M. Neural grafting in Parkinson's disease Problems and possibilities. Prog Brain Res. 2010;184:265-294.

3. Winkler C, Kirik D, Björklund A. Cell transplantation in Parkinson's disease: how can we make it work? Trends Neurosci. 2005;28(2):86-92.

4. Chen X, et al. Human bone marrow stromal cell cultures conditioned by traumatic brain tissue extracts: growth factor production. J Neurosci Res. 2002;69(5):687-691.

5. Pittenger MF, et al. Multilineage potential of adult human mesenchymal stem cells. Science. 1999;284(5411):143-147.

6. Charbord P. Bone marrow mesenchymal stem cells: historical overview and concepts. Hum Gene Ther. 2010;21(9):1045-1056

7. Li Y, et al. Intracerebral transplantation of bone marrow stromal cells in a 1-methyl-4-phenyl-1,2,3,6-tetrahydropyridine mouse model of Parkinson's disease. Neurosci Lett. 2001;316(2):67-70.

8. Park HJ, Lee PH, Bang OY, Lee G, Ahn YH. Mesenchymal stem cells therapy exerts neuroprotection in a progressive animal model of Parkinson's disease. J Neurochem. 2008;107(1):141-151.

9. Blandini F, et al. Transplantation of undifferentiated human mesenchymal stem cells protects against 6-hydroxydopamine neurotoxicity in the rat. Cell Transplant. 2010;19(2):203-217.

10. Venkataramana NK, et al. Open-labeled study of unilateral autologous bone-marrow-derived mesenchymal stem cell transplantation in Parkinson's disease. Transl Res. 2010;155(2):62-70.

11. Kuroda Y, Kitada M, Wakao S, Dezawa M. Bone marrow mesenchymal cells: how do they contribute to tissue repair and are they really stem cells? Arch Immunol Ther Exp (Warsz). 2011;59(5):369-378.

12. Wagner W, et al. Replicative senescence of mesenchymal stem cells: a continuous and organized process. PLoS One. 2008;3(5):e2213.

13. Moloney TC, et al. Survival and immunogenicity of mesenchymal stem cells from the green fluorescent protein transgenic rat in the adult rat brain. Newrorehabil Neural Repair. 2010;24(7):645-656.

14. Thompson L, Barraud P, Andersson E, Kirik D, Björklund A. Identification of dopaminergic neurons of nigral and ventral tegmental area subtypes in grafts of fetal ventral mesencephalon based on cell morphology, protein expression, and efferent projections. J Neurosci. 2005;25(27):6467-6477.

15. Mendez I, et al. Cell type analysis of functional fetal dopamine cell suspension transplants in the striatum and substantia nigra of patients with Parkinson's disease. Brain. 2005;128(pt 7):1498-1510.

16. Grealish S, et al. The A9 dopamine neuron component in grafts of ventral mesencephalon is an important determinant for recovery of motor function in a rat model of Parkinson's disease. Brain. 2010; 133(pt 2):482-495.

17. Damier P, Hirsch EC, Agid Y, Graybiel AM. The substantia nigra of the human brain. II. Patterns of loss of dopamine-containing neurons in Parkinson's disease. Brain. 1999;122(pt 8):1437-1448.

18. Wakao S, et al. Long-term observation of auto-cell transplantation in non-human primate reveals safety and efficiency of bone marrow stromal cell-derived Schwann cells in peripheral nerve regeneration. Exp Neurol. 2010;223(2):537-547.

19. Hayase $M$, et al. Committed neural progenitor cells derived from genetically modified bone marrow stromal cells ameliorate deficits in a rat model of stroke. J Cereb Blood Flow Metab. 2009; 29(8):1409-1420.

20. Dezawa M, et al. Specific induction of neuronal cells from bone marrow stromal cells and application for autologous transplantation. J Clin Invest. 2004; 113(12):1701-1710.
21. Nagane K, Kitada M, Wakao S, Dezawa M, Tabata Y. Practical induction system for dopamine-producing cells from bone marrow stromal cells using spermine-pullulan-mediated reverse transfection method. Tissue Eng Part A. 2009;15(7):1655-1665.

22. Sladek JR Jr, Collier TJ, Haber SN, Roth RH, Redmond DE Jr. Survival and growth of fetal catecholamine neurons transplanted into primate brain. Brain Res Bull. 1986;17(6):809-818.

23. Redmond DE, et al. Fetal neuronal grafts in monkeys given methylphenyltetrahydropyridine. Lancet. 1986;1(8490):1125-1127.

24. Bankiewicz KS, et al. The effect of fetal mesencephalon implants on primate MPTP-induced parkinsonism. Histochemical and behavioral studies. J Neurosurg. 1990;72(2):231-244.

25. Kordower JH, et al. Neurodegeneration prevented by lentiviral vector delivery of GDNF in primate models of Parkinson's disease. Science. 2000; 290(5492):767-773

26. Takagi $Y$, et al. Dopaminergic neurons generated from monkey embryonic stem cells function in a Parkinson primate model. J Clin Invest. 2005; 115(1):102-109.

27. Eberling JL, et al. Functional effects of AAV2-GDNF on the dopaminergic nigrostriatal pathway in parkinsonian rhesus monkeys. Hum Gene Ther. 2009; 20(5):511-518.

28. Ferri ALM, et al. Foxa 1 and Foxa2 regulate multiple phases of midbrain dopaminergic neuron development in a dosage-dependent manner. Development. 2007;134(15):2761-2769.

29. McRitchie DA, Hardman CD, Halliday GM. Cytoarchitectural distribution of calcium binding proteins in midbrain dopaminergic regions of rats and humans. J Comp Neurol. 1996;364(1):121-150.

30. Bankiewicz KS, et al. Hemiparkinsonism in monkeys after unilateral internal carotid artery infusion of 1-methyl-4-phenyl-1,2,3,6-tetrahydropyridine (MPTP). Life Sci. 1986;39(1):7-16.

31. Frey S, et al. An MRI based average macaque monkey stereotaxic atlas and space (MNI monkey space). Neuroimage. 2011;55(4):1435-1442.

32. Clarke G, et al. A one-hit model of cell death in inherited neuronal degenerations. Nature. 2000; 406(6792):195-9

33. Burbach JPH, Smidt MP. Molecular programming of stem cells into mesodiencephalic dopaminergic neurons. Trends Neurosci. 2006;29(11):601-603.

34. Lindvall $\mathrm{O}$, et al. Grafts of fetal dopamine neurons survive and improve motor function in Parkinson's disease. Science. 1990;247(4942):574-577.

35 . Freed CR, et al. Survival of implanted fetal dopamine cells and neurologic improvement 12 to 46 months after transplantation for Parkinson's disease. N Engl J Med. 1992;327(22):1549-1555.

36. Peschanski M, et al. Bilateral motor improvement and alteration of L-dopa effect in two patients with Parkinson's disease following intrastriatal transplantation of foetal ventral mesencephalon. Brain. 1994;117(pt 3):487-499.

37. Spencer DD, et al. Unilateral transplantation of human fetal mesencephalic tissue into the caudate nucleus of patients with Parkinson's disease. NEngl J Med. 1992;327(22):1541-1548.

38. Freed CR, et al. Transplantation of embryonic dopamine neurons for severe Parkinson's disease. NEngl J Med. 2001;344(10):710-719.

39. Olanow CW, et al. A double-blind controlled trial of bilateral fetal nigral transplantation in Parkinson's disease. Ann Neurol. 2003;54(3):403-414.

40. Li J-Y, Christophersen NS, Hall V, Soulet D, Brundin P. Critical issues of clinical human embryonic stem cell therapy for brain repair. Trends in Neurosciences. 2008;31(3):146-153.

41. Vinuela A, et al. Implanted reuptake-deficient or wild-type dopaminergic neurons improve $\mathrm{ON}$ L-dopa dyskinesias without OFF-dyskinesias in a rat model of Parkinson's disease. Brain. 2008; 131(pt 12):3361-3379

42. Kittappa R, Chang WW, Awatramani RB, McKay RDG. The foxa2 gene controls the birth and spontaneous degeneration of dopamine neurons in old age. PLoS Biol. 2007;5(12):e325.

43. Cooper O, et al. Differentiation of human ES and Parkinson's disease iPS cells into ventral midbrain dopaminergic neurons requires a high activity form of SHH, FGF8a and specific regionalization by retinoic acid. Mol Cell Neurosci. 2010;45(3):258-266.

44. Cai J, et al. The role of Lmx1a in the differentiation of human embryonic stem cells into midbrain dopamine neurons in culture and after transplantation into a Parkinson's disease model. Stem Cells. 2009;27(1):220-229.

45. Hargus G, et al. Differentiated Parkinson patient-derived induced pluripotent stem cells grow in the adult rodent brain and reduce motor asymmetry in Parkinsonian rats. Proc Natl Acad Sci USA. 2010; 107(36):15921-15926.

46. Sonntag K-C, et al. Context-dependent neuronal differentiation and germ layer induction of Smad4 ${ }^{-/}$and $\mathrm{Cripto}^{-/-}$embryonic stem cells. $\mathrm{Mol}$ Cell Neurosci. 2005;28(3):417-429.

47. Kriks S, et al. Dopamine neurons derived from human ES cells efficiently engraft in animal models of Parkinson's disease. Nature. 2011;480(7378):547-551.

48. O'Keeffe FE, et al. Induction of A9 dopaminergic neurons from neural stem cells improves motor function in an animal model of Parkinson's disease. Brain. 2008;131(pt 3):630-641.

49. Kuan W-L, Lin R, Tyers P, Barker RA. The importance of A9 dopaminergic neurons in mediating the functional benefits of fetal ventral mesencephalon transplants and levodopa-induced dyskinesias. Neurobiol Dis. 2007;25(3):594-608.

50. De Camilli P, Miller PE, Navone F, Theurkauf WE, Vallee RB. Distribution of microtubule-associated protein 2 in the nervous system of the rat studied by immunofluorescence. Neuroscience. 1984; 11(4):817-846.

51. Xu H, et al. Transplantation of neuronal cells induced from human mesenchymal stem cells improves neurological functions after stroke without cell fusion. J Neurosci Res. 2010;88(16):3598-3609.

52. Freeman TB, Brundin P. Important aspects of surgical methodology for transplantation in Parkinson's disease. In: Brundin P, Olanow CW, eds. Restorative Therapies in Parkinson's Disease. New York, New York, USA: Springer; 2006:131-165.

53. Dubach M, et al. Primate neostriatal neurons containing tyrosine hydroxylase: immunohistochemical evidence. Neurosci Lett. 1987;75(2):205-210.

54. Guliás B, Halldin C. New PET radiopharmaceuticals beyond FDG for brain tumor imaging. QJ Nucl Med Mol Imaging. 2012;56(2):173-190.

55. Padma MV, et al. Functional imaging of a large demyelinating lesion. J Clin Neurosci. 2005;12(2):176-178.

56. Lewis DA, Sesack SR. Dopamine systems in the primate brain. In: Bloom FE, Björklund A, Hökfelt T. The Primate Nervous System. New York, New York, USA: Elsevier; 1997:263-375.

57. Kordower JH, Liu YT, Winn S, Emerich DF. Encapsulated PC12 cell transplants into hemiparkinsonian monkeys: a behavioral, neuroanatomical, and neurochemical analysis. Cell Transplant. 1995; 4(2):155-171.

58. Luthman J, Jonsson G. Effects of the parkinsonism-inducing neurotoxin MPTP and its metabolite $\mathrm{MPP}+$ on sympathetic adrenergic nerves in mouse iris and atrium. Med Biol. 1986;64(2-3):95-102.

59. Kurlan R, Kim MH, Gash DM. Oral levodopa dose-response study in MPTP-induced hemiparkinsonian monkeys: assessment with a new rating scale for monkey parkinsonism. Mov Disord. 1991;6(2):111-118.

60. Jordan S, et al. 6-[18F]fluoro-L-m-tyrosine: metab- 
olism, positron emission tomography kinetics, and 1-methyl-4-phenyl-1,2,3,6-tetrahydropyridine lesions in primates. Brain Res. 1997;750(1-2):264-276.

61. Emborg ME, et al. Age-related declines in nigral neuronal function correlate with motor impairments in rhesus monkeys. J Comp Neurol. 1998; 401(2):253-265.

62. Saiki H, Hayashi T, Takahashi R, Takahashi J. Objective and quantitative evaluation of motor function in a monkey model of Parkinson's disease. J Neurosci Methods. 2010;190(2):198-204.
63. Någren K, Müller L, Halldin C, Swahn CG, Lehikoinen P. Improved synthesis of some commonly used PET radioligands by the use of [11C] methyl triflate. Nucl Med Biol. 1995;22(2):235-239.

64. Herzog H, et al. NEMA NU2-2001 guided performance evaluation of four Siemens ECAT PET scanners. IEEE Trans Nucl Sci. 2004;51(5):2662-2669.

65. Gunn RN, Lammertsma AA, Hume SP, Cunningham VJ. Parametric imaging of ligand-receptor binding in PET using a simplified reference region model. Neuroimage. 1997;6(4):279-287.
66. Sturgeon C. Practice guidelines for tumor marker use in the clinic. Clin Chem. 2002;48(8):1151-1159.

67. Al-Sugair A, Coleman RE. Applications of PET in lung cancer. Semin Nucl Med. 1998;28(4):303-319.

68. Nichols TE, Holmes AP. Nonparametric permutation tests for functional neuroimaging: a primer with examples. Hum Brain Mapp. 2002;15(1):1-25.

69. Smith SM, Nichols TE. Threshold-free cluster enhancement: addressing problems of smoothing, threshold dependence and localisation in cluster inference. Neuroimage. 2009;44(1):83-98. 